PHYSICAL REVIEW B 96, 205405 (2017)

\title{
Thermal conductance of metallic atomic-size contacts: Phonon transport and Wiedemann-Franz law
}

\author{
J. C. Klöckner, ${ }^{1, *}$ M. Matt, ${ }^{1}$ P. Nielaba, ${ }^{1}$ F. Pauly, ${ }^{1,2}$ and J. C. Cuevas ${ }^{1,3}$ \\ ${ }^{1}$ Department of Physics, University of Konstanz, D-78457 Konstanz, Germany \\ ${ }^{2}$ Okinawa Institute of Science and Technology Graduate University, Onna-son, Okinawa 904-0395, Japan \\ ${ }^{3}$ Departamento de Física Teórica de la Materia Condensada and Condensed Matter Physics Center (IFIMAC), \\ Universidad Autónoma de Madrid, E-28049 Madrid, Spain
}

(Received 1 September 2017; published 2 November 2017)

\begin{abstract}
Motivated by recent experiments [Science 355, 1192 (2017); Nat. Nanotechnol. 12, 430 (2017)], we present here an extensive theoretical analysis of the thermal conductance of atomic-size contacts made of three different metals, namely gold $(\mathrm{Au})$, platinum $(\mathrm{Pt})$, and aluminum $(\mathrm{Al})$. The main goal of this work is to elucidate the role of phonons in the thermal transport through these atomic contacts as well as to study the validity of the Wiedemann-Franz law, which relates the electrical and the thermal conductance. For this purpose, we have employed two different custom-developed theoretical approaches. The first one is a transport method based on density functional theory (DFT) that allows one to accurately compute the contributions of both electrons and phonons to the thermal transport in few-atom-thick contacts. The second technique is based on a combination of classical molecular dynamics (MD) simulations and a tight-binding model that enables the efficient calculation of the electronic contribution to the thermal conductance of atomic contacts of larger size. Our DFT-based calculations show that the thermal conductance of few-atom contacts of $\mathrm{Au}$ and $\mathrm{Pt}$ is dominated by electrons, with phonons giving a contribution typically below $10 \%$ of the total thermal conductance, depending on the contact geometry. For these two metals we find that the small deviations from the Wiedemann-Franz law, reported experimentally, largely stem from phonons. In the case of $\mathrm{Al}$ contacts we predict that the phononic contribution can be considerably larger with up to $40 \%$ of the total thermal conductance. We show that these differences in the phononic contribution across metals originate mainly from their distinct Debye energies. On the other hand, our MD-based calculations demonstrate that the electronic contribution to the thermal conductance follows very closely the Wiedemann-Franz law, irrespective of the material and the contact size. Finally, the ensemble of our results consistently shows that the reported observation of quantized thermal transport at room temperature is restricted to few-atom contacts of $\mathrm{Au}$, a monovalent metal in which the transport is dominated by the $s$ valence orbitals. In the case of multivalent metals like Pt and Al this quantization is statistically absent due to the fact that additional orbitals contribute to the transport with conduction channels that have intermediate transmissions between 0 and 1 , even in the case of single-atom contacts.
\end{abstract}

DOI: 10.1103/PhysRevB.96.205405

\section{INTRODUCTION}

The advent of experimental techniques like the scanning tunneling microscope and the mechanically controllable break junctions made it possible in the early 1990s to fabricate metallic atomic-size contacts all the way down to singleatom junctions and even chains of atoms [1,2]. This paved the way for investigating a large variety of charge and energy transport properties in these atomic contacts such as electrical conductance [3,4], shot noise [5-8], photocurrent [9-12], thermopower [13-16], and Joule heating [17,18], just to mention a few. The mean free path for electrons and phonons is larger than the characteristic dimensions of atomic contacts [19], even at room temperature, and all the transport properties of these nanowires are therefore dominated by quantum mechanical effects. For this reason, metallic atomic-size contacts have become an ideal playground to test basic quantum theories of charge and energy transport at the nanoscale. In fact, one can safely say that no other system has contributed so decisively to firmly establish the quantum

*Corresponding author: Jan.Kloeckner@uni-konstanz.de coherent transport picture put forward by Landauer, Büttiker, Imry, and others [1,2,20-22].

Until recently, there was a basic transport property that had not been experimentally investigated in metallic atomic-size contacts, namely the thermal conductance. This situation has now changed, and two experimental groups have finally been able to independently explore the thermal transport in atomic contacts [23,24]. In particular, Cui et al. [23] were able to measure the room-temperature thermal conductance of both $\mathrm{Au}$ and $\mathrm{Pt}$ contacts and found that in the case of Au single-atom contacts the thermal conductance is quantized in units of the universal thermal conductance quantum $\kappa_{0}=\pi^{2} k_{\mathrm{B}}^{2} T /(3 h)$, where $T$ is the absolute temperature. Before this, thermal conductance quantization had already been reported in a series of experiments, making use of different microdevices, where the heat was carried by phonons, electrons, or photons [25-29]. But in all cases sub-Kelvin temperatures were a necessary prerequisite for the observation of this quantum phenomenon. Thus, the results of Cui et al. [23] constitute the first observation of quantized thermal transport at room temperature and demonstrate the potential of these atomic contacts to reveal novel quantum effects in thermal transport. The experiments of Ref. [23] also show that the thermal conductance of $\mathrm{Pt}$ atomic-size contacts is not quantized, which 
is indeed expected in view of the fundamentally different electronic structure of this metal as compared to Au.

Another important observation of Refs. [23,24] was that the thermal conductance of atomic contacts follows closely the Wiedemann-Franz law, irrespective of the material and the contact size. This law establishes that the thermal conductance of a metal, where the heat transport is dominated by electrons, is simply proportional to its electrical conductance. The relation is known to be approximately fulfilled in macroscopic metallic wires made of standard metals, and in this case it can be explained with the help of the semiclassical Boltzmann transport equation [30]. For nanoscale devices, where the transport is fully coherent, the validity of the WiedemannFranz law requires two things: (i) The electronic transmission function must be rather smooth around the Fermi energy in an energy window of the thermal broadening $k_{\mathrm{B}} T[2,31,32]$, and (ii) the thermal transport must be largely dominated by electrons [33]. The first condition is indeed expected to be met by metallic atomic-size contacts, irrespective of the material and the contact size, as shown by numerous investigations of the conductance [1] and thermopower [13,15] of these nanowires. The second condition, which is also necessary for the observation of quantized thermal transport in Ref. [23], is by no means trivial. It is well known that the thermal conductance of macroscopic metallic wires is largely dominated by electrons, with the phonons giving a contribution that amounts to only a few percent of the total thermal conductivity [34]. However, when going from the macro- to the nanoscale, the phonon transport mechanism changes from incoherent to coherent, and it is not obvious a priori, whether the phonon contribution to the thermal transport of metallic atomic-size contacts is actually negligible. In fact, Refs. [23,24] reported slight deviations from the Wiedemann-Franz law that were actually attributed to the contribution of phonons and possibly to a small one of photons (thermal radiation) [23]. In this respect, we already showed in Ref. [23] that the contribution of the phonons to the thermal conductance of Au single-atom contacts is only around $5 \%$ of the total one, which explains the validity of the Wiedemann-Franz law and, in turn, the observation of quantized transport in these contacts. The main goal of this work is to provide a comprehensive analysis of the phonon transport in metallic atomic-size contacts made not only of $\mathrm{Au}$, but also of other relevant metals like Pt and $\mathrm{Al}$.

In this work we aim at elucidating the magnitude of the phonon contribution to the thermal conductance of metallic atomic-size contacts and to shed light on to what extent the Wiedemann-Franz law is expected to be fulfilled. For this purpose we have made use of different custom-designed theoretical techniques to describe the thermal transport in atomic junctions. In particular we have employed a full $a b$ initio, DFT-based transport method to compute the contributions of both electrons and phonons to the thermal conductance of atomic contacts made of $\mathrm{Au}, \mathrm{Pt}$, and $\mathrm{Al}$. Our calculations show that, depending on the contact size and the exact geometry, the phonons contribute by about $5 \%-10 \%$ to the total roomtemperature thermal conductance of $\mathrm{Au}$ and $\mathrm{Pt}$ contacts, which explains the small deviations from the Wiedemann-Franz law observed in Ref. [23]. Aluminum is a light metal with a high Debye energy of about $40 \mathrm{meV}$ as opposed to the 20 and 25 $\mathrm{meV}$ of $\mathrm{Au}$ and $\mathrm{Pt}$, respectively. In this case we calculate that the phonons can constitute up to $40 \%$ of the total thermal conductance contribution, depending on the geometry. These results show that phonons can in general not be ignored in the analysis of the thermal transport through metallic atomic-size contacts, especially for light metals. This is at variance with the case of macroscopic metallic wires [34].

In addition we have made use of a combination of classical MD simulations together with quantum mechanical calculations of the electronic thermal conductance based on a tight-binding model to carry out a detailed study of the validity of the Wiedemann-Franz law for $\mathrm{Au}, \mathrm{Pt}$, and $\mathrm{Al}$ contacts. Although neglecting the phonon contribution to the thermal conductance, the strength of this method is that it allows us to perform a detailed statistical analysis and to simulate both the electrical and electronic thermal conductance histograms, which enables us to establish a very direct comparison with the experiments. Our analysis confirms the expectation that the electronic thermal conductance fulfills in a very accurate manner the Wiedemann-Franz law for all three metals and irrespective of the contact size. As compared to Au and Al, we find slightly increased deviations of the order of up to $5 \%$ in the case of Pt contacts due to the fact that the transport in this metal is dominated by $d$ orbitals.

The rest of the paper is organized as follows. In Sec. II we describe the different theoretical techniques that we have used to simulate the thermal transport of metallic atomic-size contacts. In particular, we present in Sec. II A the basic formulas that describe the quantum thermal transport in these systems within the Landauer-Büttiker approach. Then, in Sec. III we discuss the main results obtained with our DFT-based transport method for the thermal conductance of $\mathrm{Au}, \mathrm{Pt}$, and $\mathrm{Al}$ few-atom contacts, with special emphasis on the relative contributions of electrons and phonons. Section IV is devoted to the analysis of the results for the electronic contribution to the thermal conductance of $\mathrm{Au}, \mathrm{Pt}$, and $\mathrm{Al}$ contacts, obtained with the help of the combined MD and tight-binding simulations. Finally, in Sec. V we summarize the main conclusions of this work.

\section{THEORETICAL APPROACHES}

In this section we describe the thermal conductance of metallic atomic-size contacts within the Landauer-Büttiker formalism for coherent quantum transport. For this purpose, we combine a number of theoretical techniques, namely the nonequilibrium Green's function (NEGF) formalism, various electronic structure methods, and classical MD simulations. We will describe these different theoretical methods in certain detail. To be precise, we will first summarize the basic formulas of the Landauer-Büttiker approach to compute the thermal conductance of a nanoscale system. Then, we will introduce our DFT-based transport approach that allows us to compute both the electronic and the phononic contributions to the thermal conductance. Finally, we will describe the MD simulations that we use to determine the geometries of the atomic contacts and how we combine the MD with a tight-binding model to compute the electronic contribution to the different transport properties of these atomic-scale contacts. 


\section{A. Thermal conductance within the Landauer-Büttiker approach}

There are two basic contributions to the thermal conductance of an atomic-size contact, namely those of electrons and phonons. The inelastic scattering lengths for electrons and phonons are clearly larger than the typical size of these metallic atomic contacts [19], even at room temperature. Thus the description of the transport properties of these systems can be performed within the Landauer-Büttiker approach for coherent transport, where one assumes that the transport, both electronic and phononic, is dominated by elastic scattering. Within this approach the contributions of electrons and phonons to the different transport properties are determined by the corresponding electronic and phononic transmission functions $\tau_{\mathrm{el}}$ and $\tau_{\mathrm{ph}}$, respectively. The electronic contribution to the linear thermal conductance $\kappa_{\mathrm{el}}$ is given by [2,35]

$$
\kappa_{\mathrm{el}}=\frac{2}{h T}\left(K_{2}-\frac{K_{1}^{2}}{K_{0}}\right),
$$

where $T$ is the absolute temperature and the $K_{n}$ coefficients are defined as

$$
K_{n}=\int_{-\infty}^{\infty}(E-\mu)^{n} \tau_{\mathrm{el}}(E)\left(-\frac{\partial f(E, T)}{\partial E}\right) d E .
$$

Here $f(E, \mu, T)=\left\{\exp \left[(E-\mu) / k_{\mathrm{B}} T\right]+1\right\}^{-1}$ is the Fermi function, and the chemical potential $\mu \approx E_{\mathrm{F}}$ is approximately given by the Fermi energy $E_{\mathrm{F}}$ of the electrodes. At this point it is important to notice that, if the electronic transmission does not strongly depend on energy in the range of some $k_{\mathrm{B}} T$ around $E_{\mathrm{F}}$, the electronic thermal conductance is approximately given by the Wiedemann-Franz law, i.e.,

$$
\kappa_{\mathrm{el}} \approx L_{0} T G \text {. }
$$

Here $L_{0}=\pi^{2} k_{\mathrm{B}}^{2} /\left(3 e^{2}\right)=2.44 \times 10^{-8} \mathrm{~W} \mathrm{~K}^{-2}$ is the socalled Lorentz number and $G$ is the electrical conductance, which we calculate as [2]

$$
G=G_{0} K_{0},
$$

with $K_{0}$ defined via Eq. (2). For low temperatures, $G$ reduces to $G=G_{0} \tau_{\mathrm{el}}\left(E_{\mathrm{F}}\right)$, where $G_{0}=2 e^{2} / h=77.48 \mu \mathrm{S}$ is the electrical conductance quantum. The test of the validity of this law in metallic atomic-size contacts is one of the central issues of this work. Notice that Eq. (3) can be rewritten as $\kappa_{\mathrm{el}}=2 \kappa_{0} \tau_{\mathrm{el}}\left(E_{\mathrm{F}}\right)$, where $\kappa_{0}=\pi^{2} k_{\mathrm{B}}^{2} T /(3 h)$ is the thermal conductance quantum, which takes the value of $\kappa_{0} \approx 0.284 \mathrm{nW} / \mathrm{K}$ at room temperature $(T=300 \mathrm{~K})$. The factor 2 in the previous equation is related to the spin degeneracy that is assumed in this work for the electronic transport. Thus, we see that in a system, where electrons dominate the quantum transport and the Wiedemann-Franz law is fulfilled, the quantization of the electrical conductance implies the quantization of the thermal conductance.

The corresponding phonon thermal conductance in the linear response regime is given by [36-38]

$$
\kappa_{\mathrm{ph}}=\frac{1}{h} \int_{0}^{\infty} E \tau_{\mathrm{ph}}(E) \frac{\partial n(E, T)}{\partial T} d E,
$$

where $n(E, T)=\left[\exp \left(E / k_{\mathrm{B}} T\right)-1\right]^{-1}$ is the Bose function, describing the phonon occupation in the electrodes. To get an idea about the order of magnitude of the phononic thermal conductance, we can express the previous equation as follows:

$$
\kappa_{\mathrm{ph}}=\kappa_{0} \int_{0}^{\infty} W_{\mathrm{ph}}(E, T) \tau_{\mathrm{ph}}(E) d E,
$$

where $\kappa_{0}$ is the thermal conductance quantum introduced above and the "window" function $W_{\mathrm{ph}}(E, T)$ is defined as

$$
W_{\mathrm{ph}}(E, T)=\frac{3}{\pi^{2}}\left(\frac{E}{k_{\mathrm{B}} T}\right)^{2}\left(-\frac{\partial n(E, T)}{\partial E}\right) .
$$

It fulfills the normalization condition,

$$
\int_{0}^{\infty} W_{\mathrm{ph}}(E, T) d E=1 .
$$

Thus, if we assume that $\tau_{\mathrm{ph}}(E)=1$ over the whole energy range, over which the function $W_{\mathrm{ph}}$ has a sizable value, then $\kappa_{\mathrm{ph}}=\kappa_{0}$. As we shall see below (c.f. Fig. 2), this condition is difficult to fulfill at room temperature due to the finite Debye energy of the different metals.

The bottom line of the previous discussion is that the description of the different transport properties, investigated in this work, requires the calculation of the electronic and phononic transmission functions. In the following subsections we will show, how we compute these functions with the help of the NEGF technique and different electronic structure methods.

\section{B. DFT-based transport calculations}

In this subsection we describe how we combine DFT with NEGF techniques to compute the electrical and thermal conductances of atomic-size contacts, taking into account the contributions of electrons and phonons. This combination makes use of the first-principles formalism developed by some of us and reported in Refs. [39,40]. In what follows, we briefly describe this formalism.

\section{Contact geometries, electronic structure, and vibrational properties}

The first step in our ab initio calculations is the construction of the atomic junction geometries. As described below in more detail, we investigate ideal geometries to simulate one-atom-thick contacts and study also the stretching of the atomic junctions to determine conductance traces in the spirit of the experiments of Ref. [23]. In both cases we make use of DFT to compute equilibrium geometries through total energy minimization and to describe their electronic structure. Vibrational properties of the optimized contacts are obtained in the framework of density functional perturbation theory (DFPT).

We use both DFT and DFPT procedures, as implemented in the quantum chemistry software package TURBOMOLE 6.5 [41-43]. In our calculations we employ the PBE exchangecorrelation functional $[44,45]$; the basis sets are def2-SV(P) for $\mathrm{Au}$ [46] and def-TZVP for Pt and Al [47]. Due to linear dependencies in bulk calculations of $\mathrm{Pt}$, we have changed the exponent of the most diffuse $s$ basis function from 0.04 a.u. ${ }^{-2}$ to 0.07 a.u. ${ }^{-2}$ and those of the most diffuse $p$ function from 0.05 a.u. $^{-2}$ to 0.08 a.u. ${ }^{-2}$. In all cases the corresponding Coulomb fitting basis is employed $[48,49]$. To ensure that 
the vibrational properties, i.e., force constants and derived vibrational energies, are accurately determined, we use very stringent convergence criteria to avoid the appearance of imaginary frequencies in the optimized contact region. Thus, total energies are converged to a precision of better than $10^{-9}$ a.u., while geometry optimizations are continued until the change of the maximum norm of the Cartesian gradient is below $10^{-5}$ a.u.

The bulk phonon properties of $\mathrm{Au}$ are determined as described in Ref. [40]. In short the dynamical matrix of the $\mathrm{Au}$ electrode is derived from those of a spherical cluster. As in the crystal the atoms of the Au cluster are positioned on an fcc lattice with a lattice constant of $a_{\mathrm{Au}}=4.08 \AA$, and force constants from the central atom to its neighbors are extracted from a sufficiently large cluster of 333 atoms. Since phonon properties are easily exchangeable between different electronic structure codes and in order to avoid the calculation of large clusters, we have changed our computational strategy for $\mathrm{Pt}$ and Al. For these two materials we use DFT and DFPT, as implemented in the plane wave code QUANTUM ESPRESSO [50] with PAW pseudopotentials taken from the PS Library [51], to calculate bulk force constants and phonon properties. For Pt we employ a grid of $24 \times 24 \times 24$ electronic $k$ points, an energy cutoff of $100 \mathrm{Ry}$, and a Marzari-Vanderbilt smearing of $0.07 \mathrm{Ry}$. The phonons are then computed on a grid of $9 \times 9 \times 9 q$ points. Similarly, for Al we utilize $24 \times 24 \times 24$ $k$ points, an energy cutoff of $100 \mathrm{Ry}$, a Marzari-Vanderbilt smearing of $0.1 \mathrm{Ry}$, and a $q$ grid of $11 \times 11 \times 11$. Similar to $\mathrm{Au}$ we consistently use the experimental lattice constants $a_{\mathrm{Pt}}=3.92 \AA$ and $a_{\mathrm{Al}}=4.05 \AA$ for the calculation of the bulk properties [19].

\section{Electron transport}

To determine the electronic structure of the atomic junctions and to compute the electronic transmission that fixes the electrical conductance and the electronic contribution to the thermal conductance within the Landauer-Büttiker approach, we use NEGFs expressed in a local nonorthogonal basis. Briefly, the local basis allows us to partition the basis states into L, C, and R ones, where L and R correspond to the left and right electrodes, respectively, while $\mathrm{C}$ corresponds to a central region including the atomic neck. Thus, the (single-particle) Hamiltonian (or Fock) matrix $\boldsymbol{H}$ can be written in the block form,

$$
\boldsymbol{H}=\left(\begin{array}{ccc}
\boldsymbol{H}_{\mathrm{LL}} & \boldsymbol{H}_{\mathrm{LC}} & \mathbf{0} \\
\boldsymbol{H}_{\mathrm{CL}} & \boldsymbol{H}_{\mathrm{CC}} & \boldsymbol{H}_{\mathrm{CR}} \\
\mathbf{0} & \boldsymbol{H}_{\mathrm{RC}} & \boldsymbol{H}_{\mathrm{RR}}
\end{array}\right) .
$$

A similar expression holds for the overlap matrix $S$. The energy-dependent electronic transmission $\tau_{\mathrm{el}}(E)$ can be expressed in terms of the Green's functions as [2]

$$
\tau_{\mathrm{el}}(E)=\operatorname{Tr}\left[\boldsymbol{\Gamma}_{\mathrm{L}}(E) \boldsymbol{G}_{\mathrm{CC}}^{\mathrm{r}}(E) \boldsymbol{\Gamma}_{\mathrm{R}}(E) \boldsymbol{G}_{\mathrm{CC}}^{\mathrm{a}}(E)\right],
$$

where the retarded Green's function is given by

$$
\boldsymbol{G}_{\mathrm{CC}}^{\mathrm{r}}(E)=\left[(E+i \eta) \boldsymbol{S}_{C C}-\boldsymbol{H}_{C C}-\boldsymbol{\Sigma}_{L}^{r}(E)-\boldsymbol{\Sigma}_{R}^{r}(E)\right]^{-1} .
$$

Here, $\eta$ is an infinitesimal positive parameter (that will be omitted hereafter), and advanced and retarded Green's functions are related by $\boldsymbol{G}_{\mathrm{CC}}^{\mathrm{a}}=\left[\boldsymbol{G}_{\mathrm{CC}}^{\mathrm{r}}\right]^{\dagger}$. The retarded selfenergies in the previous equation adopt the form,

$$
\boldsymbol{\Sigma}_{X}^{\mathrm{r}}(E)=\left(\boldsymbol{H}_{\mathrm{C} X}-E \boldsymbol{S}_{\mathrm{CX}}\right) \boldsymbol{g}_{X X}^{\mathrm{r}}(E)\left(\boldsymbol{H}_{X \mathrm{C}}-E \boldsymbol{S}_{X \mathrm{C}}\right) .
$$

The scattering rate matrices that enter the expression of the electronic transmission are given by $\boldsymbol{\Gamma}_{X}(E)=$ $i\left[\boldsymbol{\Sigma}_{X}^{\mathrm{r}}(E)-\boldsymbol{\Sigma}_{X}^{\mathrm{a}}(E)\right]$, and $\boldsymbol{g}_{X X}^{\mathrm{r}}(E)=\left(E \boldsymbol{S}_{X X}-\boldsymbol{H}_{X X}\right)^{-1}$ are the electrode Green's functions with $X=\mathrm{L}, \mathrm{R}$. Finally, it is convenient to decompose the total electronic transmission in terms of individual transmission coefficients. For this purpose we can write Eq. (10) as

$$
\tau_{\mathrm{el}}(E)=\operatorname{Tr}\left[\boldsymbol{t}_{\mathrm{el}}(E) \boldsymbol{t}_{\mathrm{el}}^{\dagger}(E)\right]=\sum_{i} \tau_{\mathrm{el}, i}(E),
$$

where $\boldsymbol{t}_{\mathrm{el}}(E)=\boldsymbol{\Gamma}_{\mathrm{L}}^{1 / 2}(E) \boldsymbol{G}_{\mathrm{CC}}^{\mathrm{r}}(E) \boldsymbol{\Gamma}_{\mathrm{R}}^{1 / 2}(E)$ is the electronic transmission amplitude matrix and $\tau_{\mathrm{el}, i}(E)$ are the eigenvalues of the transmission probability matrix $\boldsymbol{t}_{\mathrm{el}}(E) \boldsymbol{t}_{\mathrm{el}}^{\dagger}(E)$. They are known as transmission coefficients, while the corresponding eigenfunctions are referred to as conduction channels.

In order to describe the transport through the atomic contacts, we first extract $\boldsymbol{H}_{\mathrm{CC}}$ and $\boldsymbol{S}_{\mathrm{CC}}$ and the matrices $\boldsymbol{H}_{\mathrm{CX}}$ and $\boldsymbol{S}_{\mathrm{CX}}$ from a DFT calculation of an extended central cluster that includes the central wire and part of the leads. On the other hand, the electrode Green's functions $\boldsymbol{g}_{X X}^{\mathrm{r}}(E)$ are modeled as surface Green's functions of ideal semi-infinite crystals. To obtain these Green's functions, we first compute separately the electronic structure of a large spherical fcc cluster of 1415 atoms. Then we extract the bulk Hamiltonian and overlap matrix elements, and we use them to model a semi-infinite crystal that is infinitely extended perpendicular to the transport direction. The surface Green's functions are calculated from this crystal with the help of a decimation technique $[39,52]$. In this way we describe the whole system consistently within DFT, using the same nonorthogonal basis set and exchange-correlation functional everywhere.

\section{Phonon transport}

To compute the phonon transmission, appearing in Eq. (5), we use our previous work $[23,40,53,54]$ and combine DFT and NEGF techniques in the same spirit as for the electron transport. Briefly our starting point is the description of the phonons or vibrational modes of the atomic contacts within the harmonic approximation. In this approximation the phonon Hamiltonian for small displacements $\left\{Q_{\xi}\right\}$ of the atoms around their equilibrium positions $\left\{R_{\xi}^{(0)}\right\}$ adopts the form,

$$
\hat{H}=\frac{1}{2} \sum_{\xi} \hat{p}_{\xi}^{2}+\frac{1}{2 \hbar^{2}} \sum_{\xi \chi} \hat{q}_{\xi} K_{\xi \chi} \hat{q}_{\chi},
$$

where we have introduced mass-weighted displacement operators $\hat{q}_{\xi}=\sqrt{M_{\xi}} \hat{Q}_{\xi}$ and mass-scaled momentum operators $\hat{p}_{\xi}=\hat{P}_{\xi} / \sqrt{M_{\xi}}$ as conjugate variables. These variables obey the following commutation relations: $\left[\hat{q}_{\xi}, \hat{p}_{\chi}\right]=i \hbar \delta_{\xi \chi}$ and $\left[\hat{q}_{\xi}, \hat{q}_{\chi}\right]=\left[\hat{p}_{\xi}, \hat{p}_{\chi}\right]=0$. Here $\xi=(j, c)$ denotes a Cartesian component $c=x, y, z$ of atom $j$ at position $\vec{R}_{j}=\vec{R}_{j}^{(0)}+\vec{Q}_{j}$. The phonon system is characterized by its dynamical matrix $K_{\xi \chi}=\hbar^{2} \partial_{\xi \chi}^{2} E_{\mathrm{DFT}} / \sqrt{M_{\xi} M_{\chi}}$, which is the mass-weighted Hessian of the DFT total ground-state energy $E_{\mathrm{DFT}}$ with 
respect to the Cartesian atomic coordinates. These harmonic force constants are computed within DFPT.

In analogy with the electronic system above, the use of a local displacement basis enables the partitioning of the dynamical matrix into three parts, a central scattering region $\mathrm{C}$, and the two semi-infinite $\mathrm{L}$ and $\mathrm{R}$ electrodes,

$$
\boldsymbol{K}=\left(\begin{array}{ccc}
\boldsymbol{K}_{\mathrm{LL}} & \boldsymbol{K}_{\mathrm{LC}} & \boldsymbol{0} \\
\boldsymbol{K}_{\mathrm{CL}} & \boldsymbol{K}_{\mathrm{CC}} & \boldsymbol{K}_{\mathrm{CR}} \\
\mathbf{0} & \boldsymbol{K}_{\mathrm{RC}} & \boldsymbol{K}_{\mathrm{RR}}
\end{array}\right) .
$$

The energy-dependent phononic transmission $\tau_{\mathrm{ph}}(E)$ can be expressed in terms of phonon Green's functions as [37,40]

$$
\tau_{\mathrm{ph}}(E)=\operatorname{Tr}\left[\boldsymbol{D}_{\mathrm{CC}}^{\mathrm{r}}(E) \boldsymbol{\Lambda}_{\mathrm{L}}(E) \boldsymbol{D}_{\mathrm{CC}}^{\mathrm{a}}(E) \boldsymbol{\Lambda}_{\mathrm{R}}(E)\right],
$$

where $\boldsymbol{D}_{\mathrm{CC}}^{\mathrm{r}, \mathrm{a}}(E)$ are the retarded and advanced phonon Green's functions of the central region. They can be computed by solving the following Dyson equation,

$$
\boldsymbol{D}_{\mathrm{CC}}^{\mathrm{r}}(E)=\left[(E+i \eta)^{2} \mathbf{1}_{\mathrm{CC}}-\boldsymbol{K}_{\mathrm{CC}}-\boldsymbol{\Pi}_{\mathrm{L}}^{\mathrm{r}}(E)-\boldsymbol{\Pi}_{\mathrm{R}}^{\mathrm{r}}(E)\right]^{-1} .
$$

In the expression, $\eta>0$ is an infinitesimal parameter and $\boldsymbol{D}_{\mathrm{CC}}^{\mathrm{a}}(E)=\boldsymbol{D}_{\mathrm{CC}}^{\mathrm{r}}(E)^{\dagger}$. The scattering rate matrices,

$$
\boldsymbol{\Lambda}_{X}(E)=i\left[\boldsymbol{\Pi}_{X}^{\mathrm{r}}(E)-\boldsymbol{\Pi}_{X}^{\mathrm{a}}(E)\right],
$$

are related to the corresponding embedding self-energies,

$$
\boldsymbol{\Pi}_{X}^{\mathrm{r}}(E)=\boldsymbol{K}_{\mathrm{C} X} \boldsymbol{d}_{X X}^{\mathrm{r}}(E) \boldsymbol{K}_{X \mathrm{C}},
$$

which describe the coupling between the central region $\mathrm{C}$ and electrode $X$. In the expressions $\boldsymbol{d}_{X X}^{\mathrm{r}}(E)=\left[(E+i \eta)^{2} \mathbf{1}_{X X}-\right.$ $\left.\boldsymbol{K}_{\mathrm{XX}}\right]^{-1}$ is the surface Green's function of lead $X=\mathrm{L}, \mathrm{R}$, and $\Pi_{X}^{\mathrm{a}}(E)=\Pi_{X}^{\mathrm{r}}(E)^{\dagger}$.

To calculate the different parts of the dynamical matrix in Eq. (15) we follow the same strategy as in the electronic case described above. We first compute the dynamical matrix for an extended central cluster, including the atomic wire and parts of the leads. Subsequently we extract from it the matrices $\boldsymbol{K}_{\mathrm{CC}}$ and $\boldsymbol{K}_{\mathrm{C} X}$. On the other hand, the surface Green's functions of the electrodes $\boldsymbol{d}_{X X}^{\mathrm{r}}(E)$ are obtained by extracting bulk force constants either from a separate calculation of a big cluster or from a periodic bulk calculation, as discussed before in Sec. II B 1. The extracted bulk parameters are then used in combination with a decimation technique $[39,52]$ to describe the surface of a semi-infinite perfect crystal, exactly like in the electronic case.

Let us close this subsubsection by pointing out another analogy with the electronic case: We can decompose the total phononic transmission of Eq. (16) into the contribution of individual phonon transmission coefficients, $\tau_{\mathrm{ph}}(E)=$ $\sum_{i} \tau_{\mathrm{ph}, i}(E)$. Here, the coefficients $\tau_{\mathrm{ph}, i}(E)$ are the eigenvalues of $\boldsymbol{t}_{\mathrm{ph}}(E) \boldsymbol{t}_{\mathrm{ph}}^{\dagger}(E)$, where $\boldsymbol{t}_{\mathrm{ph}}(E)=\boldsymbol{\Lambda}_{\mathrm{L}}^{1 / 2}(E) \boldsymbol{D}_{\mathrm{CC}}^{\mathrm{r}}(E) \boldsymbol{\Lambda}_{\mathrm{R}}^{1 / 2}(E)$ is the phononic transmission amplitude matrix.

\section{Combination of MD simulations and a tight-binding model}

The DFT-based transport calculations are very timeconsuming and to carry out a complete statistical analysis within this $a b$ initio approach is out of reach at present. Instead we are able to perform such an analysis for the electronic transport (both for $G$ and $\kappa_{\mathrm{el}}$ ) by combining classical MD simulations of the junction formation with quantum transport calculations based on a sophisticated tight-binding model. In particular, this hybrid approach allows us to compute conductance histograms that can be directly compared with those reported experimentally. Indeed this combination has been quite successful in determining a variety of properties of these atomic-scale wires $[8,15,23,55-59]$. The disadvantage of this method, as compared to our DFT-based approach, is that at present we are not able to describe the phonon transport. But since the phonons will be shown to play a minor role in most situations, calculations of this type are extremely useful to explore, in particular, the validity of the Wiedemann-Franz law in metallic atomic-size contacts of several hundred atoms in size. In what follows, we shall describe this combined approach by presenting separately the details of the MD simulations and those related to the transport calculations based on the tight-binding model.

\section{Molecular dynamics simulations}

As mentioned above, in order to perform a thorough statistical analysis of the thermal conductance of different metallic atomic-size contacts, we carry out classical MD simulations to first determine the geometry of these contacts following our previous work [15]. The thermal conductance is then computed for the geometries determined with these MD simulations, as we explain in the next paragraph. We perform the MD simulations with the open source program package LAMMPS $[60,61]$. Within LAMMPS we use the embedded atom method with the semiempirical potentials from Ackland et al. [62] for $\mathrm{Au}$ and from Sheng et al. [63] for $\mathrm{Pt}$ and $\mathrm{Al}$ to model the interactions between atoms. It is worth stressing that these potentials account for the possibility to have an atomic coordination that differs from the bulk. In order to obtain the geometry of the atomic contacts, we start with an ideal fcc lattice, where the crystal direction $\langle 100\rangle$ lies parallel to the transport and elongation direction. In these simulations we first divide the geometry into three parts: two electrodes and a central wire, bridging the gap between them, as shown in Fig. 1. Each electrode consists of 661 atoms that are kept fixed during the simulations. The size of the electrodes is chosen such that they contain at least all those atoms that are separated from the central wire atoms by less than the cutoff radius of the interaction potentials. The central wire is made up of 563 atoms and their motion is described with Newtonian equations of motion. We assume a canonical ensemble and use the velocity Verlet integration scheme [64]. In our simulations the wires have an initial length of $0.82 \mathrm{~nm}$ for $\mathrm{Au}, 0.78 \mathrm{~nm}$ for $\mathrm{Pt}$, and $0.84 \mathrm{~nm}$ for $\mathrm{Al}$. The starting velocities of the atoms in the wire are chosen randomly with a Gaussian distribution to yield an average temperature of $T=300 \mathrm{~K}$. Because of the randomness in the initial velocity distribution, every stretching simulation is different, while a Nosé-Hoover thermostat ensures that the temperature remains fixed [64]. The time step in all our simulations is $1 \mathrm{fs}$, and in the beginning each wire is equilibrated for $0.1 \mathrm{~ns}$. Subsequently, the elongation process is simulated by separating the electrodes at a constant velocity of $0.4 \mathrm{~m} / \mathrm{s}$. During this process the geometry is recorded every $10 \mathrm{ps}$, in order to compute the transport properties. A stretching 

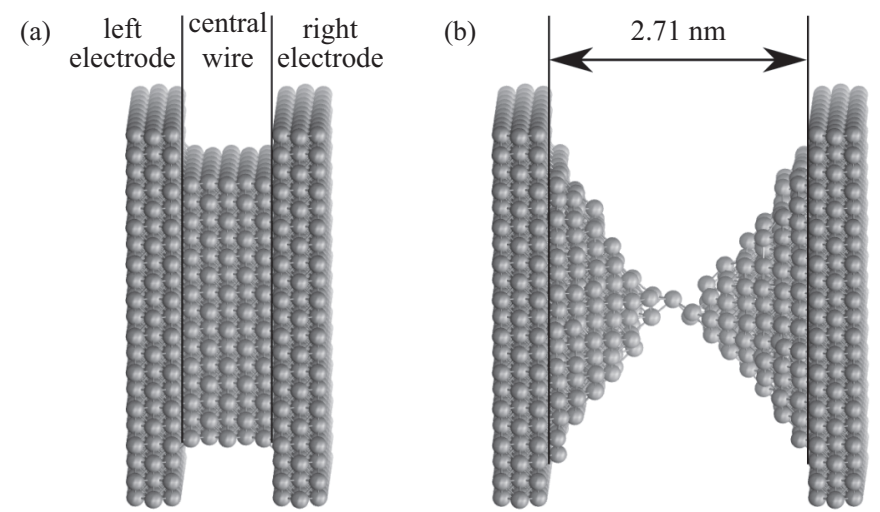

FIG. 1. (a) The initial fcc structure of the atomic contacts employed in the MD simulations. The example is for Al, where the central wire possesses a length of $0.84 \mathrm{~nm}$. We also show the partitioning of the contact into the left and right electrodes and the central wire, as used for the MD and transport calculations. (b) Example of an atomic contact of $\mathrm{Al}$ at an elongation of $1.87 \mathrm{~nm}$. Considering the initial length of the junction of $0.84 \mathrm{~nm}$, the total length of the central wire amounts to $2.71 \mathrm{~nm}$, as indicated in the panel.

process needs a total simulation time of about $4.5 \mathrm{~ns}$ until the contact breaks.

\section{Tight-binding-based transport calculations}

The geometries obtained from the MD simulations are used to compute the electronic contribution to the transport properties within the Landauer-Büttiker formalism. In this case the electronic transmission that determines both the electrical conductance and the electronic contribution to the thermal conductance is computed with the help of a tightbinding model. To be precise, we employ a nonorthogonal Slater-Koster tight-binding parametrization, which has been constructed by fitting DFT-based results for the electronic band structure and total energies of metals across the periodic table; see Refs. [65,66] for details. In this parametrization we take into account the relevant valence orbitals, which for $\mathrm{Au}$ and $\mathrm{Pt}$ include the $5 d, 6 s$, and $6 p$ orbitals, and for $\mathrm{Al}$ the $3 s, 3 p$, and $3 d$ orbitals. Moreover the hopping and overlap matrix elements in this tight-binding model are functions of the distance between the atoms, which enables us to use it with our MD simulations.

To compute the electronic transmission, we combine the tight-binding model with NEGF techniques and the formulas detailed in Sec. II A, very much like in the DFT-based calculations. Details can be found in Refs. [55,56]. Briefly, as in the MD simulations, the system is divided into three regions for the transport calculations, i.e., the two electrodes and the central wire; see Fig. 1. Because the local environment of the atoms in the central part is very different from that in the bulk, we impose a charge neutrality condition for all the atoms of the central wire [55,56], which is known to be approximately fulfilled in metallic systems. As in the DFT case the electrodes are considered to be semi-infinite perfect crystals, and their surface Green's functions are computed with the help of a decimation technique [39,52]. Again, as in the DFT-based calculations, the Green's function techniques also

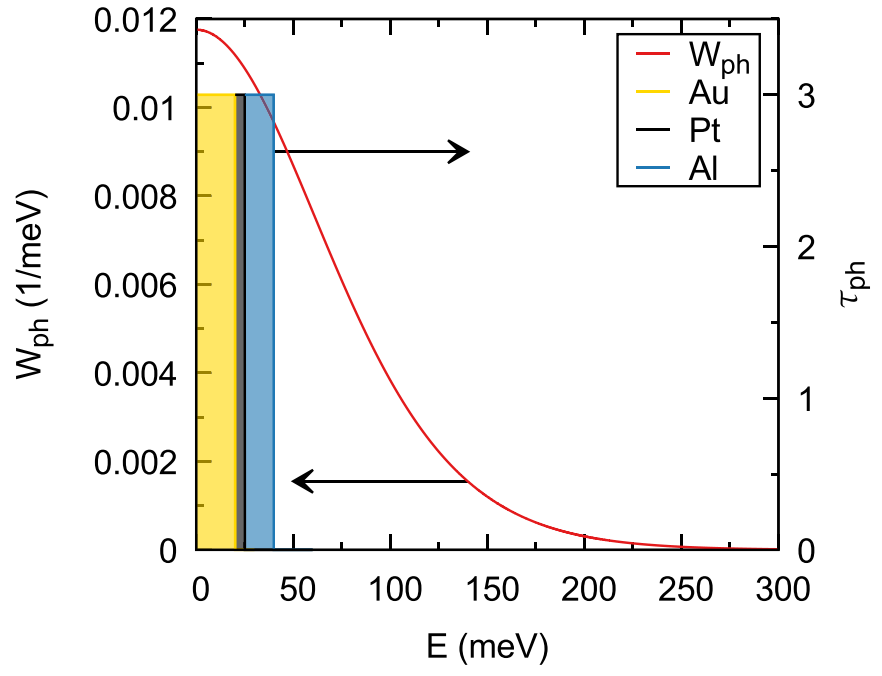

FIG. 2. Window function $W_{\mathrm{ph}}(E, T)$, defined in Eq. (7), as a function of energy at room temperature $(T=300 \mathrm{~K})$. We also show the phonon transmissions used to estimate the phononic thermal conductance of single-atom contacts of $\mathrm{Au}, \mathrm{Pt}$, and $\mathrm{Al}$. We assume for simplicity that $\tau_{\mathrm{ph}}(E)$ is equal to 3 for energies up to the Debye energy of the corresponding metal. Arrows in the plot refer to left and right vertical scales.

allow us to compute the individual transmission coefficients $\tau_{\mathrm{el}, i}(E)$ at a given energy $E$.

\section{DFT-BASED TRANSPORT RESULTS: PHONON TRANSPORT}

In this section we shall discuss the main results for the thermal conductance of metallic atomic-size contacts, obtained with our $a b$ initio, DFT-based transport method. But before doing so, it is instructive to estimate the contribution of phonons to the thermal conductance at room temperature. Let us focus, in particular, on the case of single-atom contacts. To get estimates for upper bounds, we assume that there are three phonon conduction channels. We choose the number of three, since one expects one channel for each spatial dimension. They might also be seen as a longitudinal and two transverse eigenchannels. Let us furthermore assume that the channels exhibit a perfect transparency for energies up to the corresponding Debye energy $E_{\mathrm{D}}$ of the metal: $20 \mathrm{meV}$ for $\mathrm{Au}, 25 \mathrm{meV}$ for $\mathrm{Pt}$, and $40 \mathrm{meV}$ for $\mathrm{Al}$. Thus, if we use $\tau_{\mathrm{ph}}(E)=3$ for $E \in\left[0, E_{\mathrm{D}}\right]$ in Eq. (6), as shown in Fig. 2, we obtain a room temperature phononic thermal conductance of $0.199 \mathrm{nW} / \mathrm{K}=0.7 \kappa_{0}$ for $\mathrm{Au}, 0.244 \mathrm{nW} / \mathrm{K}=0.86 \kappa_{0}$ for $\mathrm{Pt}$, and $0.378 \mathrm{nW} / \mathrm{K}=1.33 \kappa_{0}$ for $\mathrm{Al}$. Notice that the largest value arises for $\mathrm{Al}$, which is simply due to its higher Debye energy as compared to $\mathrm{Au}$ and Pt. These estimates need to be put into relation to the corresponding ones for the electronic contribution to the thermal conductance. To obtain them, we use the Wiedemann-Franz law [see Eq. (3)], and the experimentally reported values for the transmission of singleatom contacts. We extract the transmissions from the lowest peak in the electrical conductance histograms, which typically arises from single-atom contacts. In this way we expect a room temperature electronic thermal conductance of about 
TABLE I. Room-temperature values of the different transport properties for the single-atom contacts of Fig. 3. $G$ is the electrical conductance, $L_{0} T G$ is the expected result for the electronic contribution to the thermal conductance from the Wiedemann-Franz law, $\kappa_{\mathrm{el}}$ is the electronic thermal conductance, $\kappa_{\mathrm{ph}}$ is the phononic thermal conductance, and $\kappa$ is the total thermal conductance, determined as the sum of the electronic and phononic contributions.

\begin{tabular}{lccccc}
\hline \hline Metal & $G\left(G_{0}\right)$ & $L_{0} T G(\mathrm{nW} / \mathrm{K})$ & $\kappa_{\mathrm{el}}(\mathrm{nW} / \mathrm{K})$ & $\kappa_{\mathrm{ph}}(\mathrm{nW} / \mathrm{K})$ & $\kappa(\mathrm{nW} / \mathrm{K})$ \\
\hline $\mathrm{Au}$ & 1.01 & 0.577 & 0.578 & 0.051 & 0.629 \\
$\mathrm{Pt}$ & 1.83 & 1.041 & 1.098 & 0.098 & 1.196 \\
$\mathrm{Al}$ & 0.49 & 0.277 & 0.271 & 0.130 & 0.401 \\
\hline \hline
\end{tabular}

$0.568 \mathrm{nW} / \mathrm{K}=2 \kappa_{0}$ for $\mathrm{Au}$ single-atom contacts [1], roughly between $0.710 \mathrm{nW} / \mathrm{K}=2.5 \kappa_{0}$ and $1.420 \mathrm{nW} / \mathrm{K}=5 \kappa_{0}$ for $\mathrm{Pt}$ $[15,67,68]$, and something between $0.284 \mathrm{nW} / \mathrm{K}=\kappa_{0}$ and $0.568 \mathrm{nW} / \mathrm{K}=2 \kappa_{0}$ for Al [69,70]. Thus, we see that the phononic and electronic contributions could in principle be of similar order, and it is by no means obvious that phonons can be ignored in the analysis of the heat conduction in metallic atomic contacts. The quantitative determination of the relative contributions of electrons and phonons to the thermal conductance will be a central issue of the rest of this section.

Let us start our discussion of the DFT-based transport results by considering single-atom contacts of $\mathrm{Au}, \mathrm{Pt}$, and $\mathrm{Al}$. Simulations, based on both classical MD and DFT [55,56,71], show that the last conductance plateau corresponds to oneatom-thick contacts that usually feature an atomic dimer in the narrowest region before breaking. For this reason we consider the ideal dimer geometries, shown in the upper part of Fig. 3, as representative examples of single-atom contacts. For all the contacts the crystallographic $\langle 111\rangle$ direction is oriented along the transport direction. Figure 3 summarizes the transport results for the three junction materials under study, and precise values of the relevant transport properties at room temperature are summarized in Table I. Figure 3(a) displays the electronic transmission, both the total one and those of the five highest transmission coefficients, as a function of energy for the Au dimer contact. We find that the electronic transport around the Fermi energy is dominated by a single conduction channel that is almost fully open, as it has been reported before for similar geometries [4,39,72,73]. In this particular case we find that the electrical conductance is $1.01 G_{0}$, while the corresponding result for the electronic thermal conductance at room temperature is $\kappa_{\mathrm{el}}=0.577 \mathrm{nW} / \mathrm{K} \approx 2 \kappa_{0}$. This value agrees very well with the expectation from the WiedemannFranz law in Eq. (3), $L_{0} T G=0.578 \mathrm{nW} / \mathrm{K}=2 \kappa_{0}$. Turning now to the phonon contribution to the thermal transport, we show in Fig. 3(b) the corresponding phononic transmission. In this case the phononic transmission is mainly dominated by three conduction channels, which are in general partially open. (Indeed the transmission values vary between 0 and 1, depending on energy.) Notice that the transmission only differs from zero below $20 \mathrm{meV}$, which corresponds to the Au Debye energy in our calculations. Using this transmission function, we find that the phonon contribution to the thermal conductance at room temperature is $\kappa_{\mathrm{ph}}=0.051 \mathrm{nW} / \mathrm{K}=0.18 \kappa_{0}$, which is about $8 \%$ of the total thermal conductance. This value is much smaller than the upper bound provided above, because the total transmission is clearly below 3 for almost all energies, which we attribute to the mismatch between the incoming phonons and the local vibrations in the narrowest part of the contact. For completeness we also present in Fig. 3(c) the temperature dependence of the thermal conductance, including the electronic and phononic contributions, as well as the total one. Note that hereafter $\kappa=\kappa_{\mathrm{el}}+\kappa_{\mathrm{ph}}$ denotes the total thermal conductance. As one can see, and we already explained in Ref. [23], the thermal conductance of Au dimer contacts is clearly dominated by electrons for most temperatures. On the other hand, the room temperature thermal conductance quantization, as observed in Ref. [23], is a consequence of the fact that, in addition, the electrical conductance is quantized and the electronic transmission is rather smooth around the Fermi energy [see Fig. 3(a)], which implies that the WiedemannFranz law is accurately fulfilled. Note that we will refer to electrical conductance quantization as a situation, where all open transmission channels exhibit perfect transparency, i.e., $\tau_{\mathrm{el}, i}\left(E_{\mathrm{F}}\right)$ is either 1 or 0 . Let us furthermore recall that the tendency of $\mathrm{Au}$ single-atom contacts to exhibit an electrical conductance of around $1 G_{0}$ is due to the fact that the electronic transport is dominated by the $s$ valence orbitals of this metal. In general the number of conduction channels in a single-atom contact is determined by the number of valence orbitals that give a significant contribution to the density of states around the Fermi energy [4,72].

We will now discuss the results for the Pt single-atom contact shown at the top of the second column in Fig. 3. The corresponding total electronic transmission function is displayed in Fig. 3(d) along with the five most relevant transmission coefficients. As one can see, and in strong contrast with the $\mathrm{Au}$ case, there are four conduction channels that provide a sizable contribution to the transport at the Fermi energy. This is due to the fact that apart from the $s$ valence orbitals, the $d$ valence orbitals of Pt atoms also contribute to the electronic transport [15,56,74]. Moreover these orbitals yield conduction channels that are partially open, which naturally explains the lack of electrical conductance quantization in this metal $[15,67,68]$. The transmission function results in an electrical conductance of $1.83 G_{0}$, while the corresponding electronic contribution to the thermal conductance at room temperature is $1.098 \mathrm{nW} / \mathrm{K}=3.87 \kappa_{0}$. This is close to the value of $1.041 \mathrm{nW} / \mathrm{K}=3.67 \kappa_{0}$ suggested by the WiedemannFranz law. The larger deviation from this law, as compared to $\mathrm{Au}$, arises from the more pronounced energy dependence of the electronic transmission function of $\mathrm{Pt}$ around the Fermi energy, caused by the $d$ bands $[15,56,57]$. In contrast to the electronic transport, the shape of the phonon transmission of this $\mathrm{Pt}$ contact is similar to that of the $\mathrm{Au}$ contact, as visible from Fig. 3(e). This originates from the similar masses of $\mathrm{Au}$ and Pt atoms, leading to comparable Debye energies. As in the case of $\mathrm{Au}$, three to four conduction channels dominate the phonon transport in the Pt dimer contact, leading to a phonon thermal conductance of $0.098 \mathrm{nW} / \mathrm{K}=0.35 \kappa_{0}$. This value almost doubles $\kappa_{\mathrm{ph}}$ of the Au contact, but is comparable in relative terms: It also constitutes about $8 \%$ of the total thermal conductance $\kappa$ (see Table I). Figure 3(f) shows that, very much like in the case of $\mathrm{Au}$, the thermal conductance is largely dominated by the electrons at all relevant temperatures. 


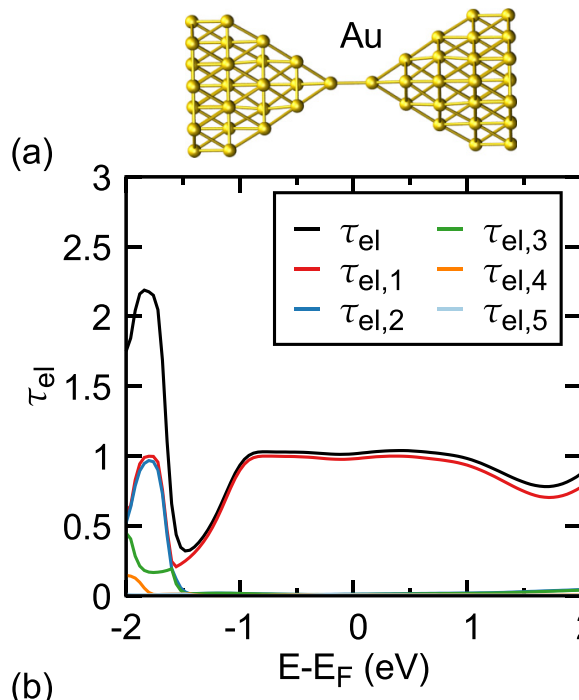

(b)

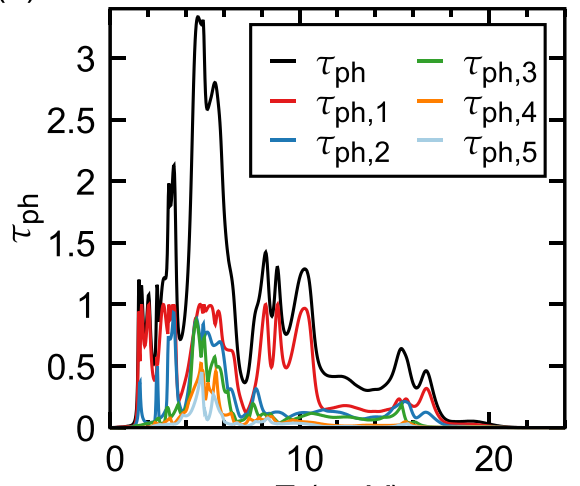

(c)

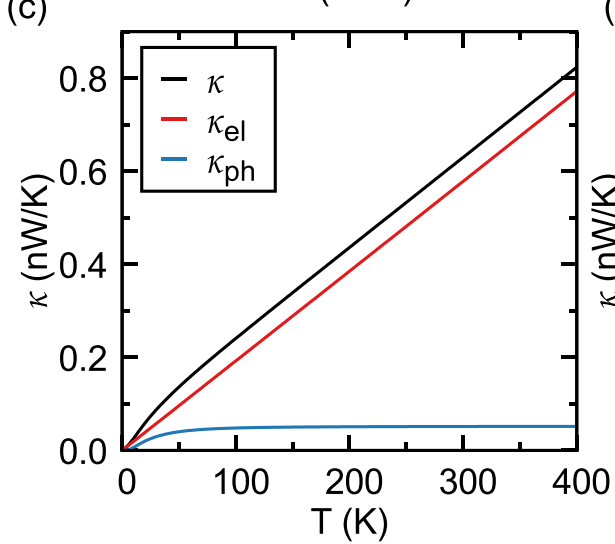

(d)
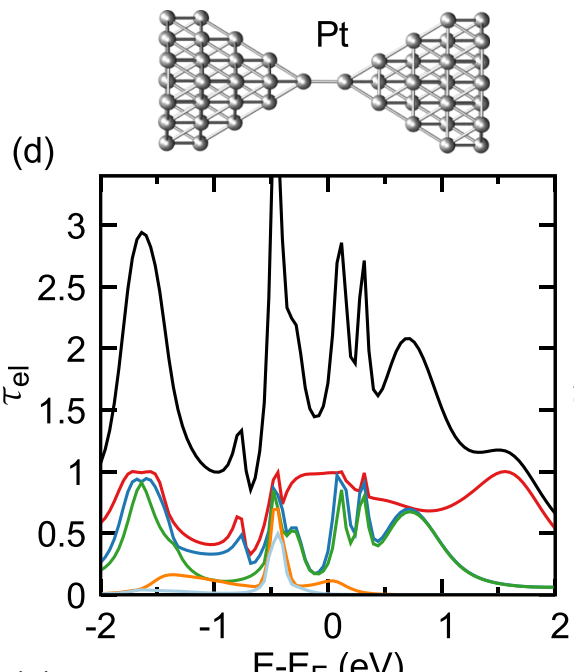

(e)

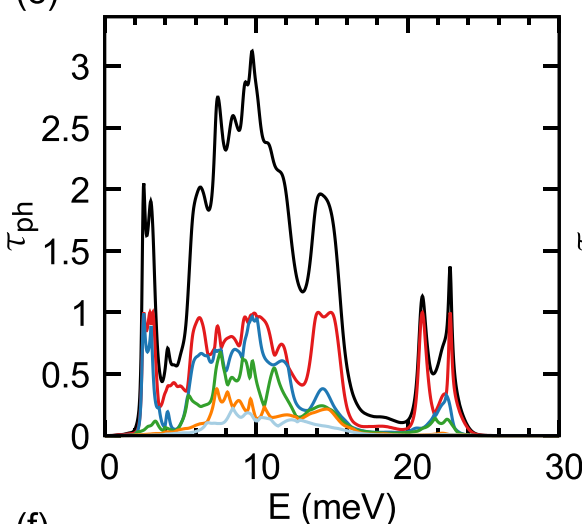

(f)

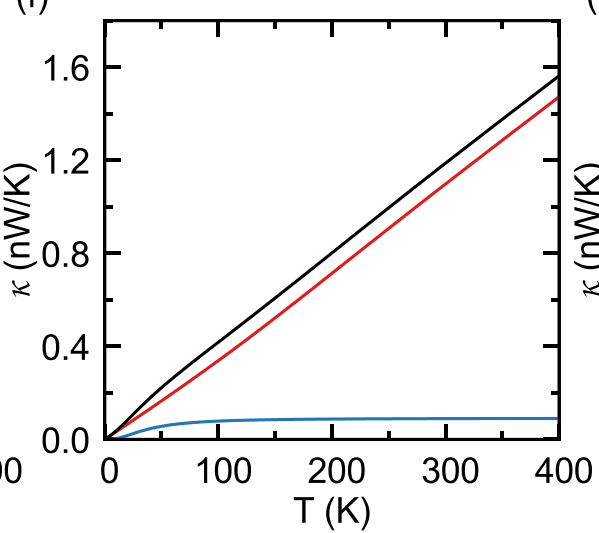

(h)

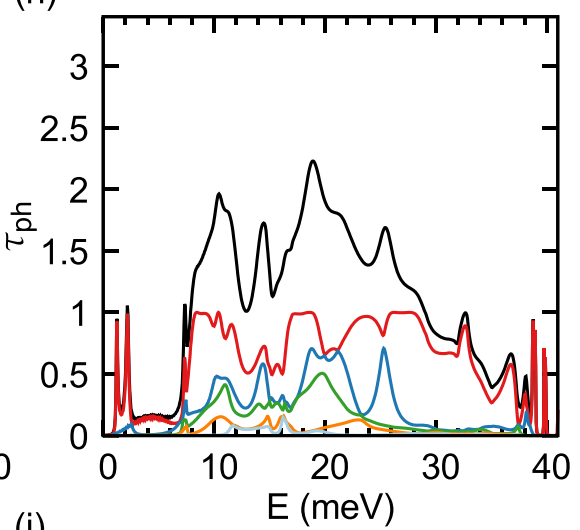

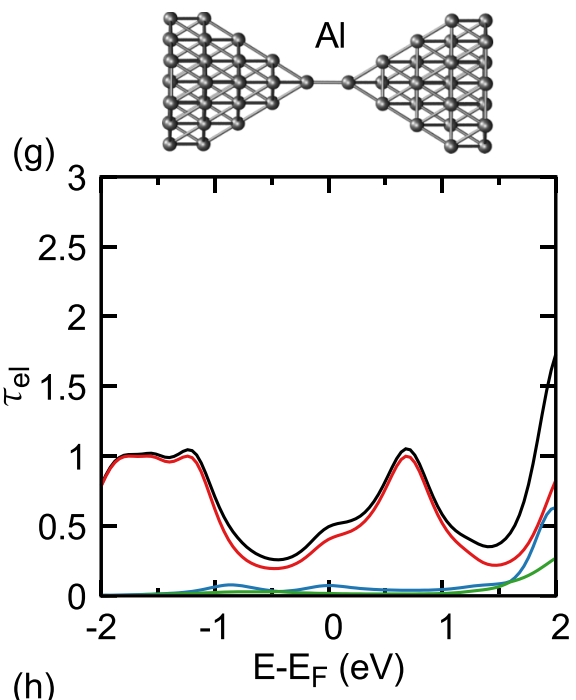

(i)

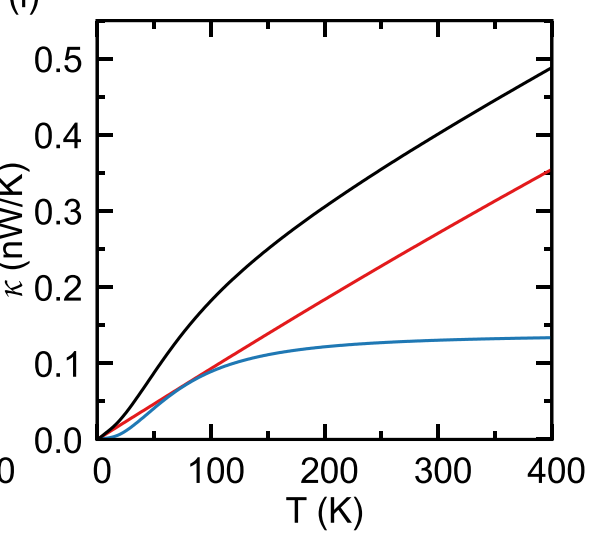

FIG. 3. (a) Electronic transmission $\tau_{\mathrm{el}}$ as a function of energy (measured with respect to the Fermi energy $E_{\mathrm{F}}$ ) for the Au single-atom contact shown above the panel. We display the total transmission as well as the five largest transmission coefficients, as indicated in the legend. (b) The corresponding phononic transmission as a function of energy. Similar to the electronic transmission we show both the total one and the largest five individual transmission coefficients. (c) Thermal conductance as a function of temperature for the Au single-atom contact with the total thermal conductance $\kappa$ resolved into electronic and phononic contributions, $\kappa_{\mathrm{el}}$ and $\kappa_{\mathrm{ph}}$, respectively. (d)-(f) The same as in panels (a)-(c) for the Pt single-atom contact shown above panel (d). (g)-(i) The same as in panels (a)-(c) for the Al single-atom contact shown above panel (g).

The lack of electrical conductance quantization results in the absence of thermal conductance quantization for single-atom contacts of Pt, as was confirmed experimentally in Ref. [23].

Let us now address the Al single-atom contact displayed above Fig. 3(g). We remark that the thermal transport in Al contacts has not been investigated experimentally so far. Aluminum is a reactive metal that is not easy to handle at room temperature, but the electronic transport through $\mathrm{Al}$ atomic contacts has been thoroughly explored at low temperatures. It is a very good example of a light metal with a Debye energy that is significantly larger than those of $\mathrm{Au}$ and $\mathrm{Pt}$. The total electronic transmission around the Fermi energy stems from two to three partially open channels, as one can see in Fig. 3(g) and has been reported both theoretically and experimentally 
in numerous occasions [4,58,70-72,75]. These observations can be understood by the contribution of both $s$ and $p$ valence orbitals of $\mathrm{Al}$ atoms [4,72]. The electrical conductance in this example is $0.49 G_{0}$, while the room temperature electronic thermal conductance is $0.277 \mathrm{nW} / \mathrm{K}=0.98 \kappa_{0}$, in very good agreement with the value of $0.271 \mathrm{nW} / \mathrm{K}=0.95 \kappa_{0}$ from the Wiedemann-Franz law. As one can see at the phonon transmission function, displayed in Fig. 3(h), the phonon transport is also dominated by three to four conduction channels, like in the $\mathrm{Au}$ and $\mathrm{Pt}$ cases, but now phonon modes up to $40 \mathrm{meV}$ participate. These additional phonon modes give rise to a phonon thermal conductance of $0.130 \mathrm{nW} / \mathrm{K}=0.46 \kappa_{0}$. This value is clearly larger than those of $\mathrm{Au}$ and $\mathrm{Pt}$. Together with the lower electrical conductance, $\kappa_{\text {ph }}$ yields now about $32 \%$ of $\kappa$ (see also Table I). Figure 3(i) shows the corresponding temperature dependence of the thermal conductance for the $\mathrm{Al}$ dimer contact. Notice that contrary to the cases of Au and Pt the phonon contribution is now of the same size as the electronic one in a broad range of temperatures up to $T=100 \mathrm{~K}$ and at room temperature it still constitutes a very significant contribution. These results suggest that a clear violation of the Wiedemann-Franz law should be observable in Al single-atom contacts, a prediction that yet awaits experimental verification.

To further study the relative contributions of electrons and phonons to the thermal conductance and to model the experiments more closely, we have used our DFT-based approach to simulate the contact formation and to compute the corresponding conductance traces. Since Au and Pt behave similarly with regard to the small contribution of phonons to $\kappa$, we concentrate in the following on a comparison of $\mathrm{Au}$ and $\mathrm{Al}$ only. We start out with atomic contacts of $\mathrm{Au}$ and $\mathrm{Al}$, and stretch or compress the geometry adiabatically. This is done by separating or by approaching the electrodes in a steplike manner and by subsequent re-optimization of the junction geometry. As the displacement step we use $0.26 \AA$ and compute $G, \kappa_{\mathrm{el}}, \kappa_{\mathrm{ph}}$, and $\kappa$ with our DFT-based transport method for the obtained equilibrium geometries.

In Fig. 4(a) we show the results of such a simulation for the $\mathrm{Au}$ contact of Fig. 3 that is grown along the $\langle 111\rangle$ direction of the fcc lattice. We started with the dimer contact represented by the second geometry in that panel, counting from the right. This geometry was compressed to obtain thicker cross sections as well as stretched to simulate the breaking of the contact. In Fig. 4(a) we show the results for $G$ and $\kappa$ at room temperature for the series of contacts, obtained following our protocol. The electrical conductance in this plot is normalized by the electrical conductance quantum $G_{0}$, while the thermal conductance is normalized by $2 \kappa_{0}$ with $T=300 \mathrm{~K}$. As one can see, both conductances proceed in a steplike manner in a succession of plateaus and abrupt jumps, related to elastic stages, where bonds are stretched and forces build up, and plastic stages, where bonds break and the accumulated tension is released. This behavior resembles the experiments [23]. In addition both conductances follow each other very closely. With the normalization used here, this means that the Wiedemann-Franz law is well obeyed. Notice also that both $G$ and $\kappa$ feature a plateau at $1 G_{0}$ and $2 \kappa_{0}$, respectively, which illustrates the tendency of $\mathrm{Au}$ single-atom contacts to exhibit quantized electronic and thermal transport, even at room temperature [23].

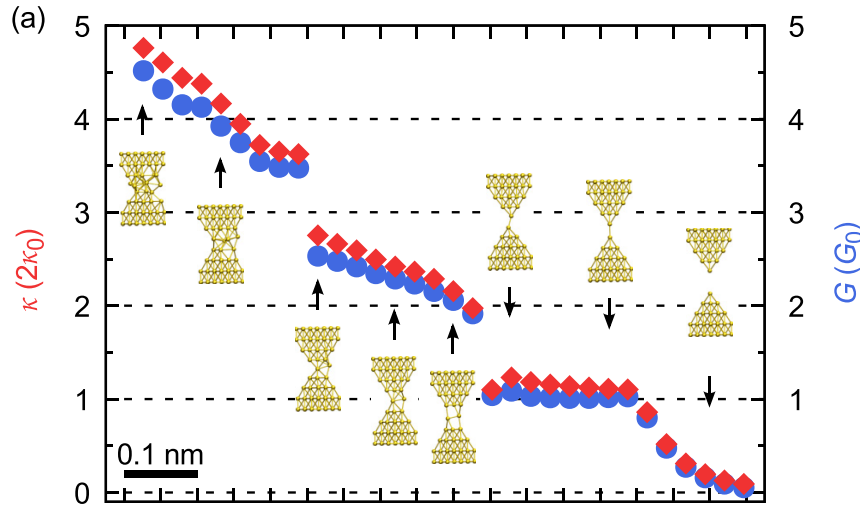

Displacement $(\mathrm{nm})$

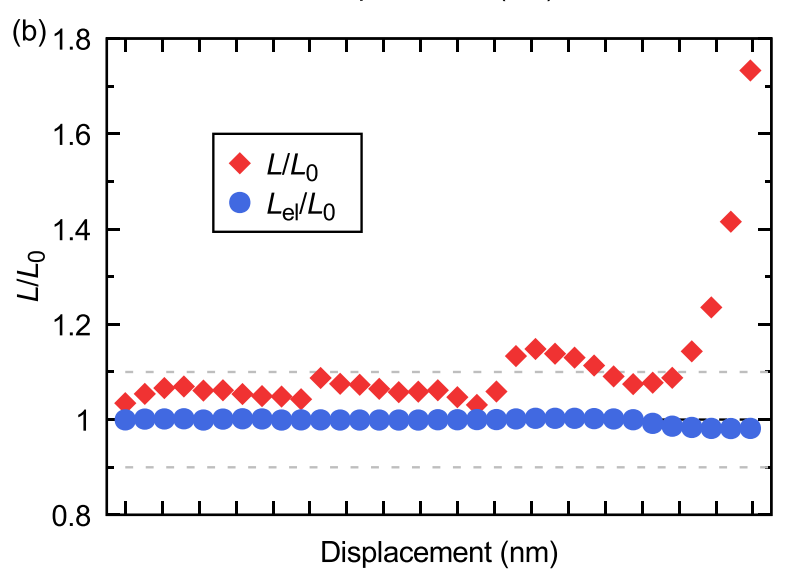

FIG. 4. (a) Electrical and thermal conductance at room temperature as a function of electrode displacement for an $\mathrm{Au}$ contact, oriented along the fcc $\langle 111\rangle$ crystallographic direction. The blue dots correspond to the results for the electrical conductance (right vertical scale), which is normalized by the electrical conductance quantum $G_{0}$. The red diamonds correspond to the total thermal conductance (left vertical scale), taking into account both the electronic and phononic contributions, and it is normalized by twice the thermal conductance quantum $\kappa_{0}$. The different geometries, shown in this panel, correspond to snapshots taken during the elongation and compression processes. (b) The corresponding Lorentz ratios, defined via Eq. (20). The red diamonds show the full ratio computed with the total thermal conductance, while the blue dots show the electronic Lorentz ratio, if the thermal conductance consists only of the electronic contribution.

To quantitatively assess the validity of the WiedemannFranz law, it is customary to define the so-called Lorentz ratio $L / L_{0}$ as follows:

$$
\frac{L}{L_{0}}=\frac{\kappa}{L_{0} T G} .
$$

Here, $\kappa=\kappa_{\mathrm{el}}+\kappa_{\mathrm{ph}}$ is the total thermal conductance due to both electrons and phonons, $L_{0}$ is the Lorentz number, and $G$ is the electrical conductance. A Lorentz ratio equal to 1 means that the measured thermal conductance agrees exactly with the expectations from the Wiedemann-Franz law, while deviations from 1 signal that this relation is violated. Such violations could be due to the contribution of phonons or they can have an electronic origin. In Fig. 4(b) we show the Lorentz ratio (red diamonds), using the results of Fig. 4(a). As one can 


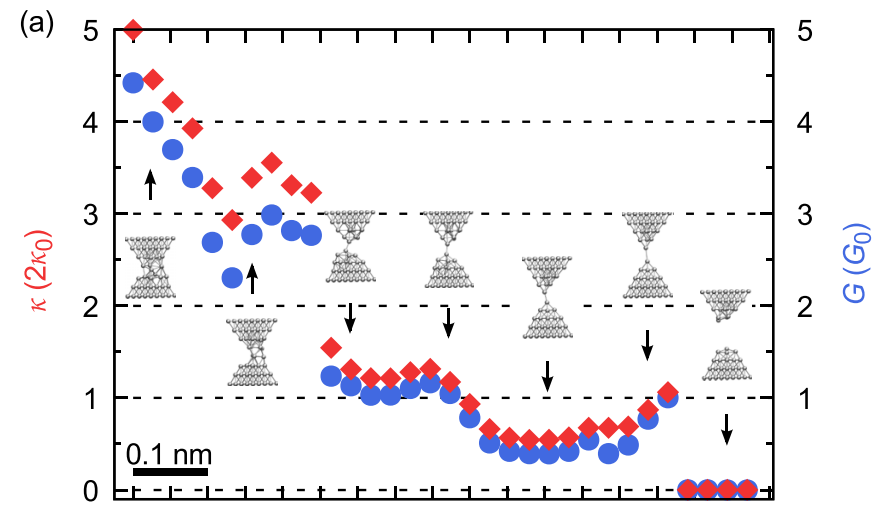

Displacement (nm)

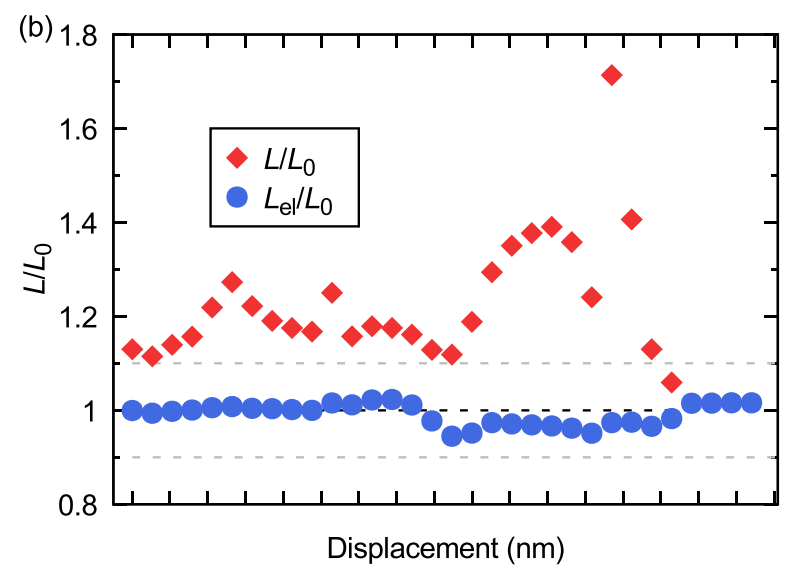

FIG. 5. The same as in Fig. 4, but for an $\mathrm{Al}$ contact.

see, there are small deviations from 1 on the order of $5 \%-$ $10 \%$ in the contact regime, depending on the exact junction geometry. These observations are in good agreement with the measurement of Ref. [23]. In order to better understand the origin of these deviations, we also show in Fig. 4(b) (blue dots) the electronic Lorentz ratio $L_{\mathrm{el}} / L_{0}=\kappa_{\mathrm{el}} /\left(L_{0} T G\right)$, constructed by replacing $\kappa$ with $\kappa_{\mathrm{el}}$ in Eq. (20). As one can see, this electronic Lorentz ratio is very close to 1 , irrespectively of the electrode displacement. This means that the deviations from the Wiedemann-Franz law are mainly due to phonons. It is also worthwhile to consider in detail the behavior of $L / L_{0}$ with the electrode displacement in Fig. 4(b). Deviations from the Wiedemann-Franz law tend to increase with larger displacements, i.e., towards smaller minimal contact cross sections. Furthermore $L / L_{0}$ exhibits a sawtoothlike shape, typically decreasing within each elastic stage. Taking into account that $L_{\mathrm{el}} / L_{0} \approx 1$ implies $L / L_{0} \approx \kappa / \kappa_{\mathrm{el}}=1+\kappa_{\mathrm{ph}} / \kappa_{\mathrm{el}}$, this means that the relative weight of $\kappa_{\mathrm{ph}}$ in $\kappa$ tends to reduce with increasing stress in the Au single-atom contacts. We attribute this to overall decreasing force constants with pulling or, in other words, a softening of interatomic bonds. Revivals of $L / L_{0}$ are seen at the points, where bonds break and the atomic contact reconfigures.

In Fig. 5 we show the results of the simulation for an $\mathrm{Al}$ atomic wire that was performed following exactly the same protocol as in the Au simulation of Fig. 4, where we use the Al dimer contact of Fig. 3 as starting geometry. As in the $\mathrm{Au}$ case, the electrical and thermal conductances proceed in a

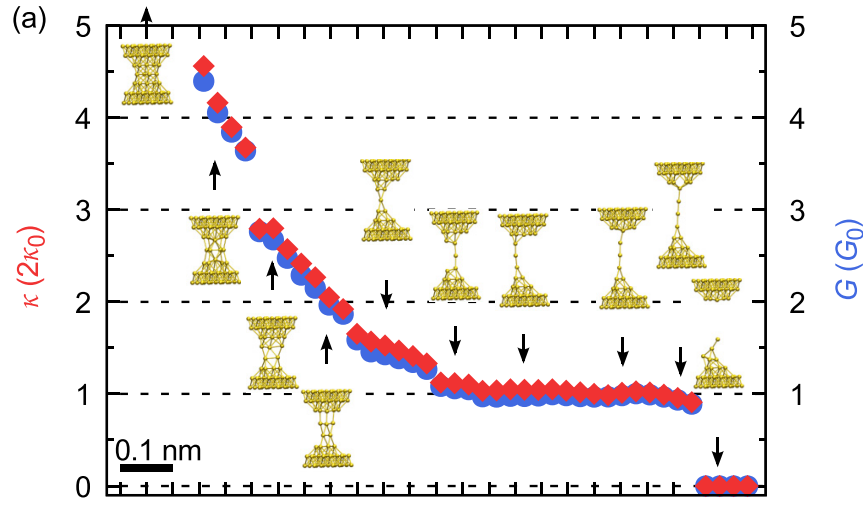

Displacement (nm)

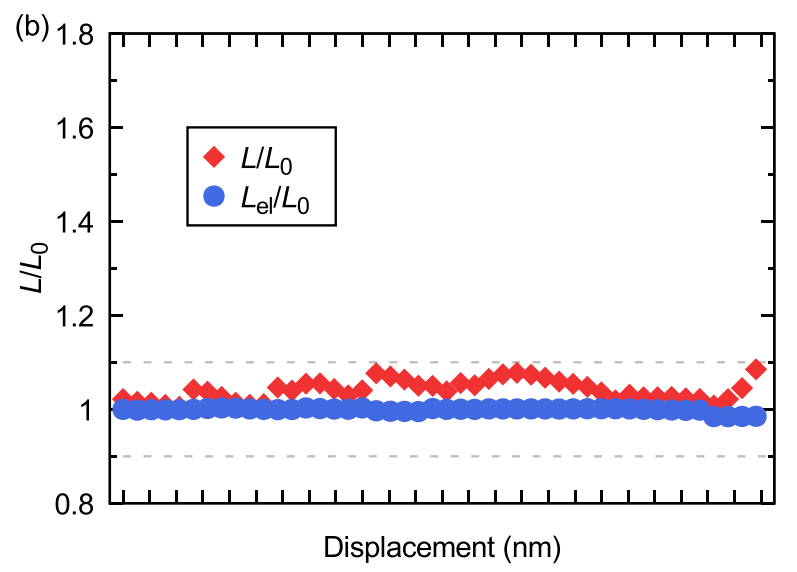

FIG. 6. The same as in Fig. 4, but for an Au contact grown along the fcc $\langle 100\rangle$ crystallographic direction. The contact was elongated, starting with the geometry shown in the upper left part of panel (a).

steplike manner with the peculiarity that most plateaus exhibit a positive slope at the end of each plateau, i.e., the conductance increases upon stretching before bonds break. This unique behavior of $\mathrm{Al}$ contacts is well known, and it has been observed in different experiments and convincingly explained $[4,70,71,75]$. The electrical and thermal conductance are correlated, but larger deviations as compared to Au are visible. This is well apparent in the Lorentz ratio, shown in Fig. 5(b), which features deviations from the Wiedemann-Franz law as large as $40 \%$ and even above. Notice also that the electronic contribution to the thermal conductance follows closely the prediction of the Wiedemann-Franz law, with deviations that are at most about $5 \%$, as can be inferred from the electronic Lorentz ratio $L_{\mathrm{el}} / L_{0}$. Thus, the larger violations of the Wiedemann-Franz relation that we find for $\mathrm{Al}$ are mainly due to the phonon contribution to the thermal transport. Our results illustrate that the phonon thermal conductance cannot always be neglected, when analyzing the thermal transport of metallic atomic-size contacts. The sawtoothlike behavior of $L / L_{0}$, discussed for Au before, is not so apparent for this $\mathrm{Al}$ contact. In any case the Lorentz ratio exhibits minima close to the displacement values, at which atomic bonds break.

It is worth stressing that we have checked that the conclusions above are not an artifact of the protocol used to simulate the contact formation or the choice of the crystallographic direction of the contact geometries. In Fig. 6 


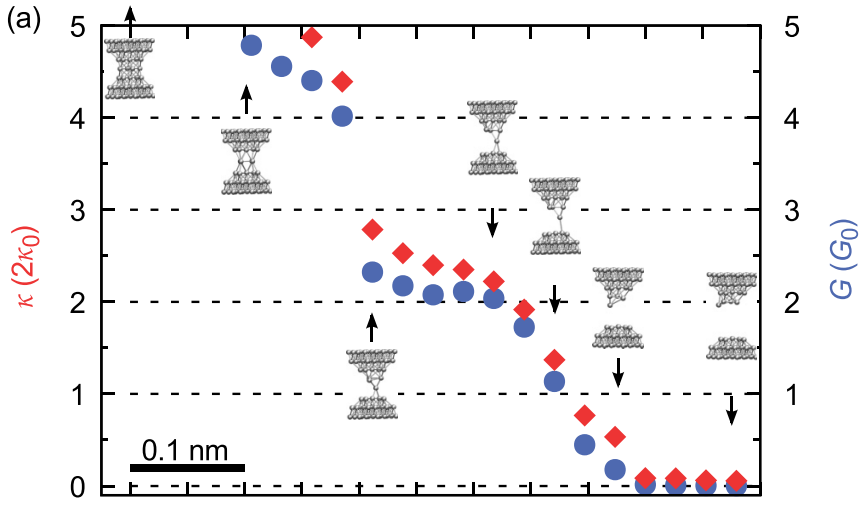

Displacement $(\mathrm{nm})$

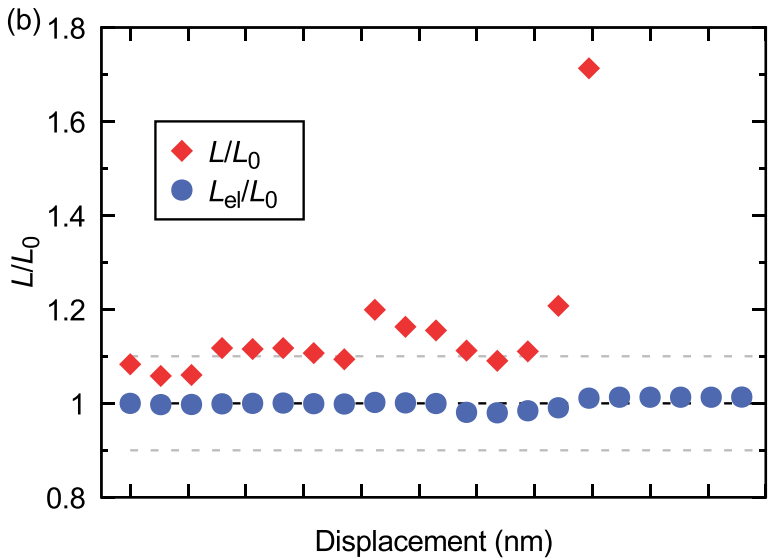

FIG. 7. The same as in Fig. 6, but for an $\mathrm{Al}$ contact.

we show an example of such tests, where we have simulated the contact formation of another $\mathrm{Au}$ atomic wire. In this case the contact is grown along the fcc $\langle 100\rangle$ direction, and we started the simulation with the geometry shown in the upper left part of Fig. 6(a). As before, we then stretched the contact progressively in steps of $0.26 \AA$. We find that all the basic observations made above about the Au contacts are reproduced here. Notably, this Au wire forms a chain with up to four atoms in length in the last stages before breaking. During the formation of this atomic chain, $G$ and $\kappa$ remain approximately quantized with values of $1 G_{0}$ and $2 \kappa_{0}$, respectively. The formation of such Au atomic chains has been reported in numerous experiments [68,76-78], and their electronic transport properties have been amply discussed in the literature [1,2]. As for the Au contact before, the plots of the Lorentz ratio show that the relative contribution of phonons to the total thermal conductance tends to decrease with increasing tension in the contact.

As a last example, we briefly discuss the stretching simulation for the Al contact depicted in Fig. 7. As in the Au case of Fig. 6, this Al contact is grown along the fcc $\langle 100\rangle$ direction and the stretching simulations were initiated with the geometry displayed in the upper left part of Fig. 7(a). There are two features of the results in Fig. 7 that we want to highlight. First of all, the last plateau before the breaking of the wire exhibits an electrical conductance of about $2 G_{0}$. It originates from a one-thick contact, where there is a monomer in the narrowest region, as opposed to the dimer realized before breaking in the previous Al simulation of Fig. 5. As we shall argue in the next section, geometries of this type are responsible for a second peak in the electrical conductance histogram of this metal close to $2 G_{0}$. The second feature worth remarking is that, as one can see in Fig. 7(b), the phonons give a slightly smaller contribution to the total thermal conductance, as compared with the example of Fig. 5, but still clearly larger than in the Au case. The sawtoothlike shape of $L / L_{0}$ as a function of electrode displacement in Fig. 7(b) is much more pronounced than in Fig. 5.

Throughout this section, we have so far only discussed the contact regime of heat transport, which has been studied experimentally $[23,24]$. But it is also interesting to examine the tunneling regime, when contacts are broken. This regime is still challenging for experimentalists, since signals are rather small and contamination of surfaces may play a crucial role [79]. Moreover, as the gap between the electrodes increases, at some point the thermal radiation via photon tunneling may give a contribution of similar order [54]. Figures 4-7 consistently show that in the tunneling regime the electronic Lorentz ratio $L_{\mathrm{el}} / L_{0}$ stays close to 1 . However, $L / L_{0}$ typically increases significantly in our calculations, meaning that the phonon thermal conductance decays more slowly with distance than the electronic part. This can be rationalized by considering that the electronic thermal conductance arises only from the overlap of electronic wave functions, which decreases exponentially with the separation of left and right junction parts. Lattice vibrations may instead couple over longer distances, because multipolar electrostatic charge contributions can, for instance, lead to an algebraic decay of $\kappa_{\mathrm{ph}}[80,81]$.

\section{ELECTRONIC THERMAL CONDUCTANCE: STATISTICAL TEST OF THE WIEDEMANN-FRANZ LAW}

The DFT simulations presented in the previous section represent the state of the art in the modeling of the thermal transport of atomic-scale systems. Ideally, one would like to perform many such simulations for even larger contacts to describe the conductance histograms for $G$ and $\kappa$ that are reported experimentally. However, these simulations are very time-consuming, and a study of conductance histograms with DFT-based methods is presently not feasible for us. Instead we employ an alternative method based on the combination of classical MD simulations and quantum transport calculations based on a tight-binding model. As described in Sec. II C, this hybrid approach allows us to determine the geometries, realized in the experiments, as well as to compute the electronic transport, i.e., $G$ and $\kappa_{\mathrm{el}}$. In particular, as shown by us previously $[15,23,55,56]$, the approach allows us to calculate conductance histograms and, in turn, to establish a very direct comparison with experiments. As compared to the adiabatic stretching processes at $T=0$, assumed in our DFT simulations above, the MD simulations offer the advantage to take also the influence of finite temperature on the formation of junction geometries into account. In this work we have used the hybrid MD and tight-binding approach to study in a systematic manner the electronic contribution to the thermal conductance in atomic contacts in order to test, whether deviations from the Wiedemann-Franz law are expected from purely electronic effects. 

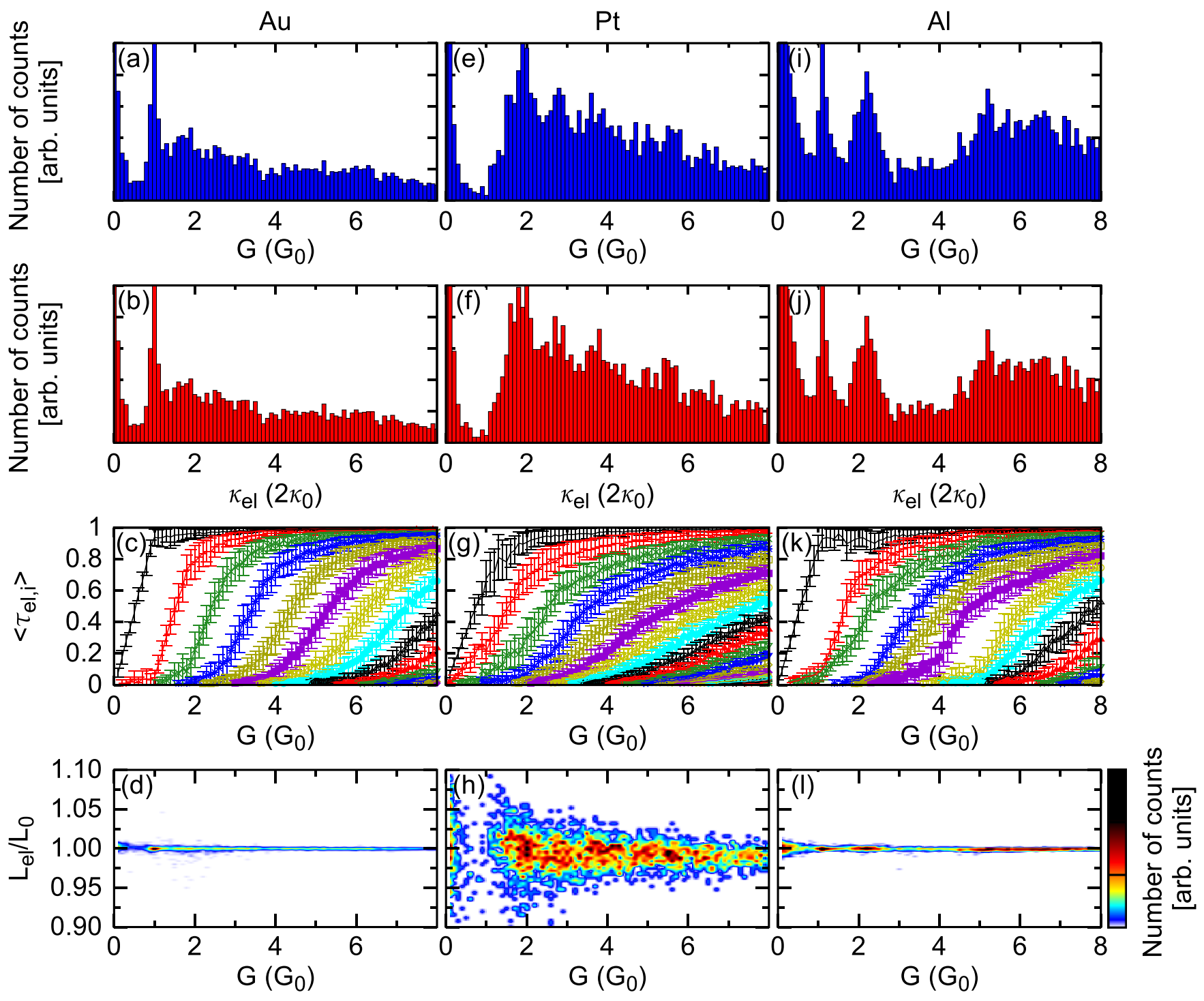

FIG. 8. (a) Electrical conductance histogram obtained from $100 \mathrm{MD}$ simulations of the stretching of Au atomic contacts at room temperature. (b) The corresponding electronic thermal conductance histogram. (c) The 20 largest electronic transmission coefficients as a function of conductance for the Au simulations. The lines correspond to the average values and the bars to the standard deviations. (d) Density plot of the electronic Lorentz ratio as a function of electrical conductance for the Au contacts. (e)-(h) The same as in panels (a)-(d), but for Pt contacts. (i)-(1) The same as in panels (a)-(d), but for Al contacts.

In Fig. 8 we summarize the results, obtained from $100 \mathrm{MD}$ simulations of the formation of $\mathrm{Au}, \mathrm{Pt}$, and $\mathrm{Al}$ atomic contacts at room temperature. In this figure we show the electrical conductance histograms, the electronic thermal conductance histograms, the evolution of electronic transmission coefficients with conductance, and the electronic Lorentz ratio. Let us stress that we have checked that 100 simulations are enough to converge the main features of the conductance histograms. We also want to point out that the results for $\mathrm{Au}$ and $\mathrm{Pt}$ were already presented in Ref. [23] and are shown here for comparison with $\mathrm{Al}$, which has not been analyzed before. The first thing to notice in Fig. 8 is the good correlation between the histograms of the electrical conductance and those of the electronic thermal conductance, which clearly indicates that the Wiedemann-Franz law is nicely fulfilled, where we ignore here of course the contribution of phonons. This is more apparent in the lower panels, where we show the electronic Lorentz ratio. As one can see, for the cases of $\mathrm{Au}$ and $\mathrm{Al}$ the deviations from 1 are very small. They are on the order of $1 \%-2 \%$, while in the case of Pt they are slightly larger and can reach up to around $5 \%$, which is consistent with our DFT results. As discussed above, these larger deviations in the case of the transition metal $\mathrm{Pt}$ are due to a more pronounced energy dependence of the electronic transmission around the Fermi energy, which in turn is due to the fact that atomic $d$ valence orbitals play a major role in the electronic transport of this metal. In summary our results show that no significant deviations from the Wiedemann-Franz law are expected from purely electronic effects, irrespective of the material or the contact size.

With respect to the conductance histograms there are several features that we want to emphasize. First of all, the $\mathrm{Au}$ 
histograms are dominated by a large peak around $1 G_{0}$ for the electrical conductance and around $2 \kappa_{0}$ for the electronic thermal conductance. Since in the Au case the phonons play a relatively minor role, this explains the observed quantized transport of $\kappa$ in Ref. [23]. The large peak for $G$ and $\kappa_{\mathrm{el}}$ in the $\mathrm{Au}$ histograms originates from single-atom contacts, where the electronic transport is dominated by a single, fully open channel, as can be deduced from Fig. 8(c). The corresponding histograms for $\mathrm{Al}$ are very interesting. They exhibit two peaks close to quantized values at $1 G_{0}$ and $2 G_{0}$, which may give the impression that the electronic transport is also quantized in $\mathrm{Al}$ few-atom contacts. However, this is actually not the case in the sense of our definition in Sec. III, which requires that transmission coefficients $\tau_{\mathrm{el}, i}$ are either 1 or 0 . As we show in Fig. $8(\mathrm{~g})$, the main contribution to those two peaks comes from contacts, where at least three partially open channels give a significant contribution to the transport. A detailed analysis shows that the lowest peak close to $1 G_{0}$ for the electrical conductance originates from one-atom-thick contacts, featuring a dimer in the narrowest part, while the second peak around $2 G_{0}$ stems mainly from contacts with only a single atom at the tightest constriction. This is indeed consistent with the observations of our DFT simulations in Figs. 5 and 7. The fact that the transmission coefficients of the open conduction channels in the last two plateaus of $\mathrm{Al}$ wires add up to a total transmission close to integer values is, in general, merely a coincidence. We do find, however, that there is a tendency for Al contacts to break, when the electrical conductance is close to $1 G_{0}$, like in the DFT simulation of Fig. 5. In those cases the transport is indeed dominated by a single conduction channel. However, along the last plateaus this is normally not the case, but we find that at least two additional channels contribute to the electronic transport. Let us remark that the multipeak structure in the electrical conductance histogram of Al contacts has been reported experimentally [69], and it is qualitatively reproduced by our MD simulations. Let us also stress that our interpretation of the lack of quantized electronic transport in $\mathrm{Al}$ contacts has been verified in great detail in experiments, where the electronic conduction channels were determined with the help of superconductivity $[4,58,75]$. Finally, in the Pt case the histogram only features a rather broad peak slightly below $2 G_{0}$ for the electrical conductance. This broad peak and the lack of quantized transport are due to the fact that even in the case of Pt single-atom contacts several conduction channels with intermediate transmissions give a significant contribution to the electronic transport; see Fig. 8(k). As explained in the previous section, this is due to the electronic structure of the transition metal $\mathrm{Pt}$, in which the atomic $d$ valence orbitals contribute decisively to both the density of states around the Fermi energy and to the electronic transport.

\section{CONCLUSIONS}

In summary, motivated by recent experiments [23,24], we have used state-of-the-art theoretical techniques to perform a systematic study of the thermal conductance of metallic atomic-size contacts made of $\mathrm{Au}, \mathrm{Pt}$, and $\mathrm{Al}$. In particular we have investigated two points of special interest, namely the contribution of phonons to the thermal transport and the validity of the Wiedemann-Franz law. Our first-principles transport calculations based on DFT show that in the case of heavy metals like $\mathrm{Au}$ and $\mathrm{Pt}$, which were experimentally investigated in Ref. [23], phonons provide a modest contribution to the thermal conductance (typically below 10\%), and this conductance is given, to a very good approximation, by the Wiedemann-Franz law. Moreover our calculations show that, while the thermal conductance of $\mathrm{Au}$ single-atom contacts is quantized due to the fact that the electronic transport is dominated by a single, fully open channel, in the case of Pt such a quantization is not present, since additional electronic conduction channels originating from the Pt $d$ bands contribute. In the case of $\mathrm{Al}$, a light metal with a much higher Debye energy, we find that the relative contribution of phonons to the thermal transport is considerably larger than for $\mathrm{Au}$ and $\mathrm{Pt}$. Indeed, it can be as large as $40 \%$ of the total thermal conductance, depending on the contact geometry. This is primarily caused by the fact that, because of the higher Debye energy of $\mathrm{Al}$, the phonon modes yield a larger contribution to the heat transport, but also due to the fact that the electrical conductance of $\mathrm{Al}$ junctions is somewhat lower than those of $\mathrm{Au}$ or $\mathrm{Pt}$ for the same contact size. Thus, our calculations show that, in general, the phonon transport cannot be ignored, when evaluating the thermal conductance of metallic atomic-size contacts. This is clearly at variance with the case of macroscopic wires, in which the phonon contribution to thermal transport is basically negligible, irrespective of the chosen (standard) metal.

Beyond the contact regime, our DFT simulations predict that phonons will contribute substantially more to thermal transport than electrons, when junctions are in the tunneling regime. While this aspect was not studied systematically, a possible explanation might be that electrostatic interactions between dipoles or higher multipoles of the electrode geometry dominate for large gaps, where the overlap of electronic wave functions of the broken sides of the contacts has decayed exponentially.

On the other hand, since our DFT results and the experiments [23] consistently suggest that electrons are more important in heat transport than phonons for metallic atomicsize contacts in the contact regime, we have employed classical MD simulations and a tight-binding model to carry out a statistical study of the purely electronic transport. In particular, we have analyzed whether the electronic contribution to the thermal conductance follows the Wiedemann-Franz law. Our results for $\mathrm{Au}, \mathrm{Al}$, and $\mathrm{Pt}$ atomic wires show that only minor deviations from the Wiedemann-Franz law are expected from the electronic contribution, irrespective of the contact size. The largest deviations, albeit modest (below 5\%), are obtained in transition metals like $\mathrm{Pt}$, where the energy dependence of the electronic transmission is more pronounced than for $\mathrm{Au}$ and $\mathrm{Al}$ due to the contribution of the $d$ orbitals. This energy dependence is also reflected in other transport properties such as the thermopower, which has been found to be larger in single-atom contacts of $\mathrm{Pt}$ than of $\mathrm{Au}$ [15]. On the other hand, the fact that the Wiedemann-Franz law is accurately fulfilled in these metallic nanowires for the electronic part provides a practical way to estimate the electronic thermal conductance from the knowledge of the electrical conductance. This, in 
turn, enables the extraction of the phonon contribution from the experimental results of the thermal conductance.

Overall our results provide deep insight into the thermal transport in metallic atomic-size contacts, one of the most important testbeds for nanoscale transport. Our results, in turn, may prove important for related systems such as single-molecule junctions, whose thermal transport properties should soon be amenable to measurement with the very same techniques that have finally enabled the exploration of the thermal conductance of metallic atomic contacts $[23,24]$.

\section{ACKNOWLEDGMENTS}

We are grateful to Pramod Reddy, Edgar Meyhofer, and Longji Cui for numerous discussions on the experimental results of Ref. [23]. J.C.K. and F.P. were financially supported by the Carl Zeiss Foundation, the Collaborative Research Center (SFB) 767 of the German Research Foundation (DFG), and the Junior Professorship Program of the Ministry of Science, Research, and the Arts Baden-Württemberg. M.M. and P.N. acknowledge funding from the SFB 767 and computer time granted by the John von Neumann Institute for Computing. J.C.C. thanks the Spanish Ministry of Economy and Competitiveness (Contract No. FIS2014-53488-P) for funding as well as the DFG and SFB 767 for sponsoring his stay at the University of Konstanz as Mercator Fellow. An important part of the numerical modeling was carried out on the computational resources of the bwHPC program, namely the bwUniCluster and the JUSTUS HPC facility.
[1] N. Agraït, A. Levy Yeyati, and J. M. van Ruitenbeek, Phys. Rep. 377, 81 (2003).

[2] J. C. Cuevas and E. Scheer, Molecular Electronics: An Introduction to Theory and Experiment, 2nd ed. (World Scientific, Singapore, 2017).

[3] J. M. Krans, J. M. van Ruitenbeek, V. V. Fisun, I. K. Yanson, and L. J. Jongh, Nature (London) 375, 767 (1995).

[4] E. Scheer, N. Agraït, J. C. Cuevas, A. Levy Yeyati, B. Ludoph, A. Martin-Rodero, G. Rubio Bollinger, J. M. van Ruitenbeek, and C. Urbina, Nature (London) 394, 154 (1998).

[5] H. E. van den Brom and J. M. van Ruitenbeek, Phys. Rev. Lett. 82, 1526 (1999).

[6] P. J. Wheeler, J. N. Russom, K. Evans, N. S. King, and D. Natelson, Nano Lett. 10, 1287 (2010).

[7] R. Chen, P. J. Wheeler, and D. Natelson, Phys. Rev. B 85, 235455 (2012).

[8] R. Vardimon, M. Matt, P. Nielaba, J. C. Cuevas, and O. Tal, Phys. Rev. B 93, 085439 (2016).

[9] D. C. Guhr, D. Rettinger, J. Boneberg, A. Erbe, P. Leiderer, and E. Scheer, Phys. Rev. Lett. 99, 086801 (2007).

[10] J. K. Viljas and J. C. Cuevas, Phys. Rev. B 75, 075406 (2007).

[11] N. Ittah, G. Noy, I. Yutsis, and Y. Selzer, Nano Lett. 9, 1615 (2009).

[12] D. R. Ward, F. Hüser, F. Pauly, J. C. Cuevas, and D. Natelson, Nat. Nanotechnol. 5, 732 (2010).

[13] B. Ludoph and J. M. van Ruitenbeek, Phys. Rev. B 59, 12290 (1999).

[14] M. Tsutsui, T. Morikawa, A. Arima, and M. Taniguchi, Sci. Rep. 3, 3326 (2013).

[15] C. Evangeli, M. Matt, L. Rincón-García, F. Pauly, P. Nielaba, G. Rubio-Bollinger, J. C. Cuevas, and N. Agraït, Nano Lett. 15, 1006 (2015).

[16] A. Ofarim, B. Kopp, T. Möller, L. Martin, J. Boneberg, P. Leiderer, and E. Scheer, Beilstein J. Nanotechnol. 7, 767 (2016).

[17] W. Lee, K. Kim, W. Jeong, L. A. Zotti, F. Pauly, J. C. Cuevas, and P. Reddy, Nature (London) 498, 209 (2013).

[18] L. A. Zotti, M. Bürkle, F. Pauly, W. Lee, K. Kim, W. Jeong, Y. Asai, P. Reddy, and J. C. Cuevas, New J. Phys. 16, 015004 (2014).

[19] N. W. Ashcroft and N. Mermin, Solid State Physics (Harcourt, Orlando, 1976).
[20] M. Büttiker, Y. Imry, R. Landauer, and S. Pinhas, Phys. Rev. B 31, 6207 (1985).

[21] M. Büttiker, Phys. Rev. Lett. 57, 1761 (1986).

[22] Y. Imry and R. Landauer, Rev. Mod. Phys. 71, S306 (1999).

[23] L. Cui, W. Jeong, S. Hur, M. Matt, J. C. Klöckner, F. Pauly, P. Nielaba, J. C. Cuevas, E. Meyhofer, and P. Reddy, Science 355, 1192 (2017).

[24] N. Mosso, U. Drechsler, F. Menges, P. Nirmalraj, S. Karg, H. Riel, and B. Gotsmann, Nat. Nanotechnol. 12, 430 (2017).

[25] K. Schwab, E. A. Henriksen, J. M. Worlock, and M. L. Roukes, Nature (London) 404, 974 (2000).

[26] O. Chiatti, J. T. Nicholls, Y. Y. Proskuryakov, N. Lumpkin, I. Farrer, and D. A. Ritchie, Phys. Rev. Lett. 97, 056601 (2006).

[27] M. Meschke, W. Guichard, and J. P. Pekola, Nature (London) 444, 187 (2006).

[28] S. Jezouin, F. D. Parmentier, A. Anthore, U. Gennser, A Cavanna, Y. Jin, and F. Pierre, Science 342, 601 (2013).

[29] M. Partanen, K. Y. Tan, J. Govenius, R. E. Lake, M. K. Makela, T. Tanttu, and M. Mottonen, Nat. Phys. 12, 460 (2016).

[30] J. M. Ziman, Electrons and Phonons: The Theory of Transport Phenomena in Solids (Oxford University Press, Oxford, 2001).

[31] P. N. Butcher, J. Phys.: Condens. Matter 2, 4869 (1990).

[32] H. van Houten, L. W. Molenkamp, C. W. J. Beenakker, and C. T. Foxon, Semicond. Sci. Technol. 7, B215 (1992).

[33] Indeed, the Wiedemann-Franz law establishes a relation between the electronic contribution to the thermal conductance and the corresponding electrical conductance. Thus, for this law to be verified experimentally, it is necessary that electrons dominate the thermal transport over any other kind of heat carriers.

[34] A. Jain and A. J. H. McGaughey, Phys. Rev. B 93, 081206 (2016).

[35] U. Sivan and Y. Imry, Phys. Rev. B 33, 551 (1986).

[36] L. G. C. Rego and G. Kirczenow, Phys. Rev. Lett. 81, 232 (1998).

[37] N. Mingo and L. Yang, Phys. Rev. B 68, 245406 (2003).

[38] T. Yamamoto and K. Watanabe, Phys. Rev. Lett. 96, 255503 (2006).

[39] F. Pauly, J. K. Viljas, U. Huniar, M. Häfner, S. Wohlthat, M. Bürkle, J. C. Cuevas, and G. Schön, New J. Phys. 10, 125019 (2008).

[40] M. Bürkle, T. J. Hellmuth, F. Pauly, and Y. Asai, Phys. Rev. B 91, 165419 (2015). 
[41] Computer code TURBOMOLE 6.5, TURBOMOLE GmbH Karlsruhe, http://www.turbomole.com. TURBOMOLE is a development of University of Karlsruhe and Forschungszentrum Karlsruhe 1989-2007, TURBOMOLE GmbH since 2007.

[42] P. Deglmann, F. Furche, and R. Ahlrichs, Chem. Phys. Lett. 362, 511 (2002).

[43] P. Deglmann, K. May, F. Furche, and R. Ahlrichs, Chem. Phys. Lett. 384, 103 (2004).

[44] J. P. Perdew and Y. Wang, Phys. Rev. B 45, 13244 (1992).

[45] J. P. Perdew, K. Burke, and M. Ernzerhof, Phys. Rev. Lett. 77, 3865 (1996).

[46] F. Weigend and R. Ahlrichs, Phys. Chem. Chem. Phys. 7, 3297 (2005).

[47] A. Schäfer, C. Huber, and R. Ahlrichs, J. Chem. Phys. 100, 5829 (1994).

[48] K. Eichkorn, F. Weigend, O. Treutler, and R. Ahlrichs, Theor. Chem. Acc. 97, 119 (1997).

[49] F. Weigend, Phys. Chem. Chem. Phys. 8, 1057 (2006).

[50] P. Giannozzi, S. Baroni, N. Bonini, M. Calandra, R. Car, C. Cavazzoni, D. Ceresoli, G. L. Chiarotti, M. Cococcioni, I. Dabo, A. Dal Corso, S. Fabris, G. Fratesi, S. de Gironcoli, R. Gebauer, U. Gerstmann, C. Gougoussis, A. Kokalj, M. Lazzeri, L. Martin-Samos, N. Marzari, F. Mauri, R. Mazzarello, S. Paolini, A. Pasquarello, L. Paulatto, C. Sbraccia, S. Scandolo, G. Sclauzero, A. P. Seitsonen, A. Smogunov, P. Umari, and R. M. Wentzcovitch, J. Phys.: Condens. Matter 21, 395502 (2009).

[51] PsLibrary, https://github.com/dalcorso/pslibrary (accessed on 08/28/2017).

[52] F. Guinea, C. Tejedor, F. Flores, and E. Louis, Phys. Rev. B 28, 4397 (1983).

[53] J. C. Klöckner, M. Bürkle, J. C. Cuevas, and F. Pauly, Phys. Rev. B 94, 205425 (2016).

[54] J. C. Klöckner, R. Siebler, J. C. Cuevas, and F. Pauly, Phys. Rev. B 95, 245404 (2017).

[55] M. Dreher, F. Pauly, J. Heurich, J. C. Cuevas, E. Scheer, and P. Nielaba, Phys. Rev. B 72, 075435 (2005).

[56] F. Pauly, M. Dreher, J. K. Viljas, M. Häfner, J. C. Cuevas, and P. Nielaba, Phys. Rev. B 74, 235106 (2006).

[57] F. Pauly, J. K. Viljas, M. Bürkle, M. Dreher, P. Nielaba, and J. C. Cuevas, Phys. Rev. B 84, 195420 (2011).

[58] C. Schirm, M. Matt, F. Pauly, J. C. Cuevas, P. Nielaba, and E. Scheer, Nat. Nanotechnol. 8, 645 (2013).

[59] R. Chen, M. Matt, F. Pauly, P. Nielaba, J. C. Cuevas, and D. Natelson, J. Phys.: Condens. Matter 26, 474204 (2014).
[60] LAMMPS Molecular Dynamics Simulator, http://lammps. sandia.gov (accessed on 08/28/2017).

[61] S. Plimpton, J. Comput. Phys. 117, 1 (1995).

[62] G. J. Ackland, G. Tichy, V. Vitek, and M. W. Finnis, Philos. Mag. A 56, 735 (1987).

[63] H. W. Sheng, M. J. Kramer, A. Cadien, T. Fujita, and M. W. Chen, Phys. Rev. B 83, 134118 (2011).

[64] D. Frenkel and B. Smit, Understanding Molecular Simulation (Academic Press, San Diego, 2002).

[65] M. J. Mehl and D. A. Papaconstantopoulos, Phys. Rev. B 54, 4519 (1996).

[66] M. J. Mehl and D. A. Papaconstantopoulos, Computational Materials Science, edited by C. Fong (World Scientific, Singapore, 1998).

[67] S. K. Nielsen, Y. Noat, M. Brandbyge, R. H. M. Smit, K. Hansen, L. Y. Chen, A. I. Yanson, F. Besenbacher, and J. M. van Ruitenbeek, Phys. Rev. B 67, 245411 (2003).

[68] R. H. M. Smit, C. Untiedt, G. Rubio-Bollinger, R. C. Segers, and J. M. van Ruitenbeek, Phys. Rev. Lett. 91, 076805 (2003).

[69] A. I. Yanson and J. M. van Ruitenbeek, Phys. Rev. Lett. 79, 2157 (1997).

[70] J. C. Cuevas, A. Levy Yeyati, A. Martin-Rodero, G. R. Bollinger, C. Untiedt, and N. Agraït, Phys. Rev. Lett. 81, 2990 (1998).

[71] P. Jelínek, R. Pérez, J. Ortega, and F. Flores, Phys. Rev. B 68, 085403 (2003).

[72] J. C. Cuevas, A. Levy Yeyati, and A. Martin-Rodero, Phys. Rev. Lett. 80, 1066 (1998).

[73] G. Rubio-Bollinger, C. de las Heras, E. Bascones, N. Agraït, F. Guinea, and S. Vieira, Phys. Rev. B 67, 121407(R) (2003).

[74] C. Sirvent, J. G. Rodrigo, S. Vieira, L. Jurczyszyn, N. Mingo, and F. Flores, Phys. Rev. B 53, 16086 (1996).

[75] E. Scheer, P. Joyez, D. Esteve, C. Urbina, and M. H. Devoret, Phys. Rev. Lett. 78, 3535 (1997).

[76] A. I. Yanson, G. Rubio Bollinger, H. E. van den Brom, N. Agraït, and J. M. van Ruitenbeek, Nature (London) 395, 783 (1998).

[77] H. Ohnishi, Y. Kondo, and K. Takayanagi, Nature (London) 395, 780 (1998).

[78] V. Rodrigues, T. Fuhrer, and D. Ugarte, Phys. Rev. Lett. 85, 4124 (2000).

[79] L. Cui, W. Jeong, V. Fernández-Hurtado, J. Feist, F. J. GarciaVidal, J. C. Cuevas, E. Meyhofer, and P. Reddy, Nat. Commun. 8, 14479 (2017).

[80] S. Xiong, K. Yang, Y. A. Kosevich, Y. Chalopin, R. D’Agosta, P. Cortona, and S. Volz, Phys. Rev. Lett. 112, 114301 (2014).

[81] V. Chiloyan, J. Garg, K. Esfarjani, and G. Chen, Nat. Commun. 6, 6755 (2015). 